\title{
COUPLED MODE EQUATIONS AND GAP SOLITONS IN HIGHER DIMENSIONS
}

\author{
TOMÁŠ DOHNAL ${ }^{1}$ AND LISA WAHLERS ${ }^{2}$
}

\begin{abstract}
We study waves-packets in nonlinear periodic media in arbitrary $(d)$ spatial dimension, modeled by the cubic Gross-Pitaevskii equation. In the asymptotic setting of small and broad waves-packets with $N \in \mathbb{N}$ carrier Bloch waves the effective equations for the envelopes are first order coupled mode equations (CMEs). We provide a rigorous justification of the effective equations. The estimate of the asymptotic error is carried out in an $L^{1}$-norm in the Bloch variables. This translates to a supremum norm estimate in the physical variables. In order to investigate the existence of gap solitons of the $d$-dimensional CMEs, we discuss spectral gaps of the CMEs. For $N=4$ and $d=2$ a family of time harmonic gap solitons is constructed formally asymptotically and numerically. Moving gap solitons have not been found for $d>1$ and for the considered values of $N$ due to the absence of a spectral gap in the standard moving frame variables.
\end{abstract}

Key-words: waves-packets, coupled mode equations, periodic media, gap soliton, approximation error, Gross-Pitaevskii

MSC: 35Q55, 35Q60, 35L71, 41A60

\section{INTRODUCTION}

One of the intriguing properties of nonlinear dispersive equations is the possibility of coherent spatially localized waves. We study such waves in the form of waves-packets in periodic media of arbitrary dimension. There are two standard asymptotic scalings of such waves-packets: waves-packets with a single carrier Bloch wave and those with multiple $(N)$ carrier Bloch waves with different group velocities. In the former case the waves-packet propagates at a velocity asymptotically close to the carrier group velocity $\left(v_{g}\right)$ and the waves-packet envelope can be (in generic situations) described by the constant coefficient nonlinear Schrödinger equation [4, 10, 7]. This is based on Taylor-expanding the band structure at the carrier wavevector to second order and using the moving frame variable with the velocity $v_{g}$. In the latter case $N$ carriers with the same temporal frequency $\omega_{0}$ are used and the band structure is expanded only to first order producing a system of $N$ first order amplitude equations for the $N$ envelopes, so called coupled mode equations (CMEs) [2, 16, 10, 3, 6, 14. In the one dimensional case the CMEs have a family of solitary waves parametrized by the velocity $v \in(-1,1)$ (after a rescaling). As these solitary waves exist in the spectral gap of the CMEs, they are called gap solitons. In a special case (corresponding to an infinitesimal contrast in the periodicity in (1.1)) there is even an explicit gap soliton family. In the general case only numerical representations of gap solitons have been found [5, 6]. An important question is whether also in the higher dimensional ( $d$-dimensional) CMEs such a family of gap solitons exists. These gap solitons would then approximate coherent waves-packets of the original model with a range of velocities in a $d$-dimensional interval and with frequencies asymptotically close to $\omega_{0}$. Such a tunability is interesting from a phenomenological as well as an applied point of view.

Date: October 12, 2018. 
As a prototypical model we consider in this paper the $d$-dimensional periodic Gross-Pitaevskii equation with the cubic nonlinearity

$$
\mathrm{i} \partial_{t} u+\Delta u-(V(x)+\varepsilon W(x)) u-\sigma(x)|u|^{2} u=0, x \in \mathbb{R}^{d}, t \in \mathbb{R},
$$

where

$$
V, \sigma \in C\left(\mathbb{R}^{d}, \mathbb{R}\right) \text { are } 2 \pi \mathbb{Z}^{d}-\text { periodic, }
$$

$\varepsilon>0$ is a small parameter, and

$$
W(x)=\sum_{m=-m_{*}}^{m_{*}} a_{m} e^{\mathrm{i} l^{(m)} \cdot x}
$$

is a real periodic function specified in (A4) below. Precise regularity conditions on $V$ and $\sigma$ appear in (A5) and are needed for the justification of amplitude equations in Sec. 6. Our analysis can be carried over to other cubically nonlinear wave models with only small modifications. An example is the wave equation $\partial_{t}^{2} u-\Delta u+(V(x)+\varepsilon W(x)) u+\sigma(x) u^{3}=0$.

Our formal asymptotic ansatz for an $N$-mode waves-packet solution to (1.1) is

$$
u^{\mathrm{app}}(x, t):=\varepsilon^{1 / 2} \sum_{j=1}^{N} A_{j}(\varepsilon x, \varepsilon t) p_{j}(x) e^{\mathrm{i}\left(k^{(j)} \cdot x-\omega_{0} t\right)},
$$

where $p_{j}(x) e^{\mathrm{i}\left(k^{(j)} \cdot x-\omega_{0} t\right)}, j=1, \ldots, N$ are the carrier Bloch waves, see Sec. 1.1. The functions $p_{j}(x):=p_{n_{j}}\left(x, k^{(j)}\right)$ are eigenfunctions of the Bloch eigenvalue problem (1.7) and the wavevectors $k^{(j)}$ lie in the Brillouin zone

$$
\mathbb{B}:=\left(-\frac{1}{2}, \frac{1}{2}\right]^{d}
$$

corresponding to the $2 \pi \mathbb{Z}^{d}$ periodicity. The asymptotic parameter $\varepsilon$ in the ansatz is the same as the $\varepsilon$ in equation (1.1). The role of the periodic perturbation $\varepsilon W$ of the linear potential is to provide for linear coupling among the modes. This is explained below and in more detail in Sec. 1.1. Note that if the periods of $V$ and $W$ are not commensurate, the potential $V+\varepsilon W$ is quasiperiodic. In our asymptotic result this aspect plays, however, no role.

Under assumptions (A1), (A2), and (A4) one can formally derive the following coupled mode equations (CMEs) for the envelopes $A_{j}, j=1, \ldots, N$. For details see Sec. 1.1.

$$
\mathrm{i}\left(\partial_{T} A_{j}+v_{g}^{(j)} \cdot \nabla_{X} A_{j}\right)+\sum_{r=1}^{N} \kappa_{j r} A_{r}+N_{j}(\vec{A})=0, j=1, \ldots, N,
$$

where for $j, r \in\{1, \ldots, N\}$

$$
\begin{aligned}
N_{j}(\vec{A}) & :=\sum_{\substack{(\alpha, \beta, \gamma) \in\{1, \ldots, N\}^{3} \\
k^{(\alpha)}-k^{(\beta)}+k(\gamma) \in k^{(j)}+\mathbb{Z}^{d}}} \gamma_{j}^{(\alpha, \beta, \gamma)} A_{\alpha} \bar{A}_{\beta} A_{\gamma}, \\
\gamma_{j}^{(\alpha, \beta, \gamma)} & :=-\int_{\mathbb{T}} \sigma(x) p_{\alpha}(x) \overline{p_{\beta}}(x) p_{\gamma}(x) \overline{p_{j}}(x) e^{\mathrm{i}\left(k^{(\alpha)}-k^{(\beta)}+k^{(\gamma)}-k^{(j)}\right) \cdot x} \mathrm{~d} x, \\
\kappa_{j r} & :=-\sum_{\begin{array}{c}
m \in\left\{-m_{*}, \ldots, m_{*}\right\} \\
k^{(r)}+l^{(m)} \in k^{(j)}+\mathbb{Z}^{d}
\end{array}} a_{m} \int_{\mathbb{T}} e^{\mathrm{i}\left(k^{(r)}+l^{(m)}-k^{(j)}\right) \cdot x} p_{r}(x) \overline{p_{j}}(x) \mathrm{d} x,
\end{aligned}
$$

and where $\mathbb{T}:=\mathbb{R}^{d} /\left(2 \pi \mathbb{Z}^{d}\right)$ is a $d$-dimensional torus. Due to the assumptions on $a_{m}$ and $l^{(m)}$ in (A4) the matrix $\kappa=\left(\kappa_{j r}\right)_{j, r=1}^{N}$ is Hermitian.

In the three dimensional case CMEs have been formally derived in [3] as envelope equations for the Maxwell equations. In [9] the $d$-dimensional CMEs 1.3 have been analyzed. However, because the authors start with $(1.1)$ with $W \equiv 0$, the coupling coefficient matrix $\kappa$ vanishes. 
With $\kappa=0$ there is no spectral gap of the linear part of CMEs because the dispersion relation of the CMEs is then given by the four hyperplanes $K \mapsto v_{g}^{(j)} \cdot K, K \in \mathbb{R}^{d}$. No (exponentially) localized solitary waves of CMEs are thus expected with $\kappa=0$. We show in Sec. 3 that at least for $N=4$ there are $\kappa \neq 0$ such that a spectral gap exists and standing solitary waves can be constructed.

One of the main contributions of the paper is a rigorous justification of $(1.3)$ as valid amplitude equations for the waves-packets (1.2) in (1.1). In [9] such a justification was performed for $W \equiv 0$. The authors work in the space $H_{\varepsilon}^{s}\left(\mathbb{R}^{d}\right):=\left\{f \in L^{2}\left(\mathbb{R}^{d}\right): \int_{\mathbb{R}^{d}}\left(1+|\varepsilon k|^{2}\right)|\hat{f}(k)|^{2} \mathrm{~d} k<\infty\right\}$. Because of the loss of $d / 2$ powers of $\varepsilon$ when evaluating the $L^{2}\left(\mathbb{R}^{d}\right)$ norm of a function $f(\varepsilon \cdot)$, the authors are forced to work with additional higher order terms in the asymptotic expansion of the waves-packet. In order to invert the linear operator near the concentration $k$-points of the new correction terms the authors of [9] impose a closed mode system condition (a condition on the terms generated by a repeated application of the nonlinearity on the ansatz). In our approach we work in an $L^{1}$ space in the Bloch variables similarly to [6], where the one dimensional case was considered. The $L^{1}$-approach for the justifictaion of coupled mode equations was used already in [16] and [14, where the case of periodicity with an infinitesimal contrast (i.e. $V \equiv 0$ in (1.1)) was considered and the analysis was carried out in Fourier variables. The one dimensional CMEs for the infinitesimal contrast were justified also in [10, with estimates in Sobolev norms. In [6] the one dimensional case with $V \neq 0$ is treated and Bloch variables are needed -similarly to the current paper. By an analog of the Riemann-Lebesgue Lemma we can estimate the supremum of the error in $x$-variables in terms of the $L^{1}$ norm in Bloch variables. Via this approach we avoid working with high order terms in the asymptotics.

We use the following assumptions.

(A1) $k^{(1)}, \ldots, k^{(N)} \in \mathbb{B}$ and $n_{1}, \ldots, n_{N} \in \mathbb{N}$ are such that $\left(n_{1}, k^{(1)}\right), \ldots,\left(n_{N}, k^{(N)}\right)$ are pairwise distinct and $\omega_{n_{j}}\left(k^{(j)}\right), j=1, \ldots, N$ are eigenvalues of of (1.7) with

$$
\omega_{n_{1}}\left(k^{(1)}\right)=\omega_{n_{2}}\left(k^{(2)}\right)=\cdots=\omega_{n_{N}}\left(k^{(N)}\right)=\omega_{0} \in \mathbb{R} .
$$

If $k^{\left(j_{1}\right)}=k^{\left(j_{2}\right)}=\cdots=k^{\left(j_{I}\right)}$ for some $\left\{j_{1}, \ldots, j_{I}\right\} \subset\{1, \ldots, N\}$, then the corresponding eigenfunctions $p_{n_{j_{1}}}\left(x, k^{\left(j_{1}\right)}\right), \ldots, p_{n_{j_{I}}}\left(x, k^{\left(j_{I}\right)}\right)$ are $L^{2}(\mathbb{T})$-orthogonal.

(A2) $v_{g}^{(j)}$ is twice continuously differentiable at $k^{(j)}$ for each $j=1, \ldots, N$.

(A3) $\left|\omega_{n}(l)-\omega_{0}\right|>\delta>0$ for all $(n, l) \in(\mathbb{N} \times J) \backslash\left\{\left(n_{1}, k^{(1)}\right), \ldots,\left(n_{N}, k^{(N)}\right)\right\}$ with $J$ defined in (6.12).

(A4) $W(x)=\sum_{m=-m_{*}}^{m_{*}} a_{m} e^{\mathrm{il}(m)} \cdot x$, where $m_{*} \in \mathbb{N}$, the vectors $l^{(m)} \in \mathbb{R}^{d}, m=-m_{*}, \ldots, m_{*}$ are pairwise distinct, and

$$
a_{-m}=\overline{a_{m}}, l^{(-m)}=-l^{(m)} \text { for all } m \in\left\{0,1, \ldots, m_{*}\right\} .
$$

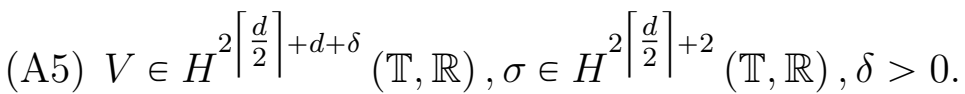

(A6) For each $j \in\{1, \ldots, N\}$ there is $L>0$, such that

$$
\left\|p_{n_{j}}(\cdot, k)-p_{n_{j}}\left(\cdot, k^{(j)}\right)\right\|_{L^{2}(\mathbb{T})} \leqslant L\left|k-k^{(j)}\right|
$$

for all $k$ in a neighborhood of $k^{(j)}$.

For $a \in \mathbb{R}$ we denote by $\lceil a\rceil$ the smallest integer larger than or equal to $a$.

Note that if at each $k^{(j)}$ the eigenvalue $\omega_{n_{j}}\left(k^{(j)}\right)$ is simple, then (1.4) holds automatically, see [13, Sec. VII.2].

The potential $\varepsilon W$ is introduced in order to linearly couple the modes of the ansatz (1.2). In detail, two modes $e^{\mathrm{i} k^{(j)} \cdot x} p_{n_{j}}\left(x, k^{(j)}\right)$ and $e^{\mathrm{i} k^{(\tilde{j})} \cdot x} p_{n_{\tilde{j}}}\left(x, k^{(\tilde{j})}\right)$ with $j, \tilde{j} \in\{1, \ldots, N\}$ are coupled if

$$
k^{(j)}-k^{(\tilde{j})}=l^{(m)}+\mu \text { for some } m \in\left\{-m_{*}, \ldots, m_{*}\right\}, \mu \in \mathbb{Z}^{d},
$$


and

$$
a_{m} \int_{\mathbb{T}} e^{\mathrm{i}\left(k^{(\tilde{j})}+l^{(m)}-k^{(j)}\right) \cdot x} p_{\tilde{j}}(x) \overline{p_{j}}(x) \mathrm{d} x \neq 0 .
$$

The first condition implies that

$$
e^{\mathrm{i} k^{(\tilde{j})} \cdot x} e^{\mathrm{i} l^{(m)} \cdot x}=e^{\mathrm{i} k^{(j)} \cdot x} \psi(x),
$$

where $\psi$ is $2 \pi \mathbb{Z}^{d}$-periodic. For details see Section 1.1 .

Our justification result is

Theorem 1. Assume (A1)-(A6) and let $\vec{A}$ be a solution of 1.3$)$ with $\hat{A}_{j} \in C\left(\left[0, T_{0}\right], L_{s_{A}}^{1}\left(\mathbb{R}^{d}\right)\right.$ $\left.\cap L^{2}\left(\mathbb{R}^{d}\right)\right), \partial_{T} \hat{A}_{j} \in C\left(\left[0, T_{0}\right], L^{1}\left(\mathbb{R}^{d}\right)\right)$ for some $T_{0}>0$, all $j=1 \ldots, N$ and some $s_{A}>$ $2\left\lceil\frac{d}{2}\right\rceil+d+2$. Then there are constants $c>0$ and $\varepsilon_{0}>0$, such that if $u(\cdot, 0)=u^{a p p}(\cdot, 0)$ with $u^{\text {app }}$ given by (1.2), then for all $\varepsilon \in\left(0, \varepsilon_{0}\right)$ the solution $u$ of $(1.1)$ satisfies $u(x, t) \rightarrow 0$ as $|x| \rightarrow \infty$ and

$$
\left\|u(\cdot, t)-u^{a p p}(\cdot, t)\right\|_{C_{b}^{0}} \leqslant c \varepsilon^{3 / 2} \text { for all } t \in\left[0, \varepsilon^{-1} T_{0}\right] .
$$

The rest of the paper is organized as follows. In Sec. 1.1 we review the Bloch eigenvalue problem and provide a brief formal derivation of the CMEs (1.3). In Sec. 2 an asymptotic approximation of standing (time harmonic) gap solitons of the CMEs is constructed for frequencies in a spectral gap and asymptotically close to the spectrum. Sec. 3 studies the dispersion relation and the gap structure of CMEs with $N$ odd and $N=2,4$ and finds that for $d \geqslant 2$ a necessary condition for the existence of a standing gap soliton is $N \in\{4,6,8, \ldots\}$. An example with a gap for $N=4$ is produced and in Sec. 4 a standing gap soliton is found numerically. The question of moving gap solitons is addressed in Sec. 5. It is shown that for $d \geqslant 2$ and $N \leqslant 4$ no spectral gap is present in the CMEs in moving frame variables. Finally, Sec. 6 presents a proof of Theorem 1, i.e. a rigorous justification of CMEs (1.3) as amplitude equations for (1.1).

\subsection{The Band Structure and a Formal Derivation of the Coupled Mode Equations.}

The bounded elementary solutions of the linear part of (1.1) at $\varepsilon=0$ are the Bloch waves

$$
p_{n}(x, k) e^{\mathrm{i}\left(k \cdot x-\omega_{n}(k) t\right)}, k \in \mathbb{B}, n \in \mathbb{N},
$$

where $\left(\omega_{n}(k), p_{n}(x, k)\right), n \in \mathbb{N}$ are eigenpairs of the Bloch eigenvalue problem

$$
\begin{aligned}
& \mathcal{L}(x, k) p_{n}(x, k)=\omega_{n}(k) p_{n}(x, k), x \in \mathbb{T}, \\
& \mathcal{L}(x, k):=-|\nabla+\mathrm{i} k|^{2}+V(x)=-\left(\Delta+2 \mathrm{i} k \cdot \nabla-\left|k^{2}\right|\right)+V(x) .
\end{aligned}
$$

The graph $\left(k, \omega_{n}(k)\right)_{n \in \mathbb{N}}$ is called the band structure. The eigenfunctions $p_{n}(\cdot, k)$ are automatically $2 \pi$-periodic in each coordinate. Because of the self-adjointness of $\mathcal{L}(\cdot, k)$ the eigenfunction sequence $\left(p_{n}(\cdot, k)\right)_{n \in \mathbb{N}}$ can be chosen $L^{2}(\mathbb{T})$-orthonormal.

We have the following asymptotics of the eigenvalues. There exist constants $c_{1}, c_{2}>0$ such that for all $n \in \mathbb{N}, k \in \mathbb{B}$

$$
c_{1} n^{2 / d} \leqslant\left|\omega_{n}(k)\right| \leqslant c_{2} n^{2 / d}
$$

, see [12, p.55].

The group velocity of the Bloch wave $(1.6)$ is $\nabla \omega_{n}(k)$, which has got also the following integral representation obtained by differentiating (1.7) in $k$

$$
\nabla \omega_{n}(k)=-2\left\langle(\nabla+\mathrm{i} k) p_{n}(\cdot, k), p_{n}(\cdot, k)\right\rangle_{L^{2}(\mathbb{T})} .
$$

For the operator $-\Delta+V: L^{2}\left(\mathbb{R}^{d}\right) \rightarrow L^{2}\left(\mathbb{R}^{d}\right)$ it is $\operatorname{spec}(-\Delta+V)=\cup_{n \in \mathbb{N}} \omega_{n}(\mathbb{B})$, see Chapter 3 in 8 . 
Figure 1 shows the band structure $k \mapsto\left(\omega_{n}(k)\right)_{n \in \mathbb{N}}($ with $k \in \mathbb{B})$ for the example $V(x)=$ $\cos \left(x_{1}\right) \cos \left(x_{2}\right)$. Only the first six eigenvalues $k \mapsto \omega_{n}(k), n=1, \ldots, 6$ are plotted. To illustrate the asymptotic ansatz 1.2 , we choose the example $N=4$ with $k^{(1)}=(-0.2,-0.4), k^{(2)}=$ $(0.2,-0.4), k^{(3)}=-k^{(1)}, k^{(4)}=-k^{(2)}$ and $n_{1}=\cdots=n_{4}=4$. With the potential $V(x)=$ $\cos \left(x_{1}\right) \cos \left(x_{2}\right)$ we have $\omega_{0}:=\omega_{4}\left(k^{(1)}\right)=\cdots=\omega_{4}\left(k^{(4)}\right) \approx 0.9942$. The points $k^{(1)}$ and $k^{(2)}$ are marked in Fig. 1. In Fig. 2 (a) we plot $\left|p_{1}\right|$, where $p_{j}:=p_{n_{j}}\left(\cdot, k^{(j)}\right), j=1, \ldots, 4$. The other Bloch functions are related by $p_{2}(x)=p_{1}\left(-x_{1}, x_{2}\right), p_{3}=\overline{p_{1}}$ and $p_{4}=\overline{p_{2}}$. Finally, Fig. 2 (b) shows $\left|u^{\text {app }}(\cdot, 0)\right|$ for the above setting and with $A_{1}(X, 0)=\cdots=A_{4}(X, 0)=e^{-|X|^{2}}, \varepsilon=0.12$.

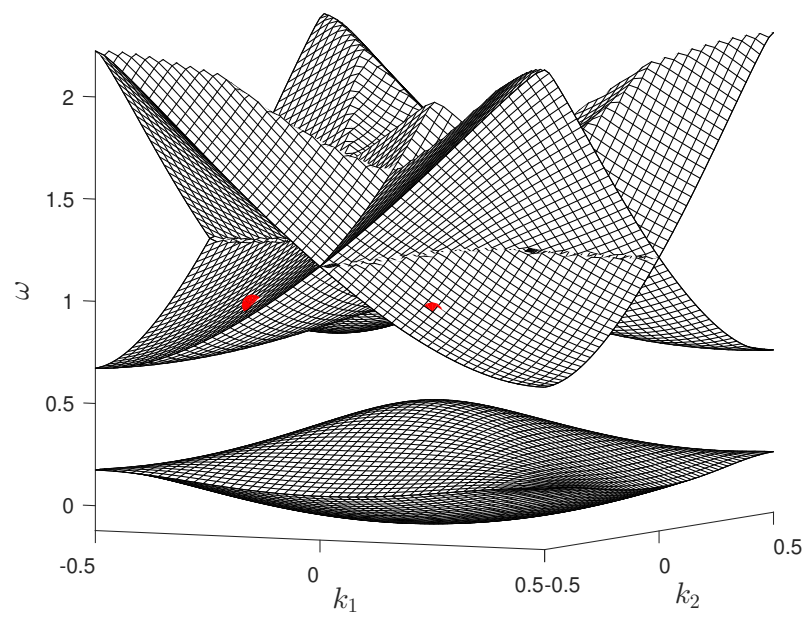

Figure 1. The band structure of (1.6) for $V(x)=\cos \left(x_{1}\right) \cos \left(x_{2}\right)$. The points $\left(k^{(1)}, \omega_{0}\right)$ and $\left(k^{(2)}, \omega_{0}\right)$ are marked by red dots. The points $k^{(3)}$ and $k^{(4)}$ satisfy $k^{(3)}=-k^{(1)}$ and $k^{(4)}=-k^{(2)}$.

(b)

(a)

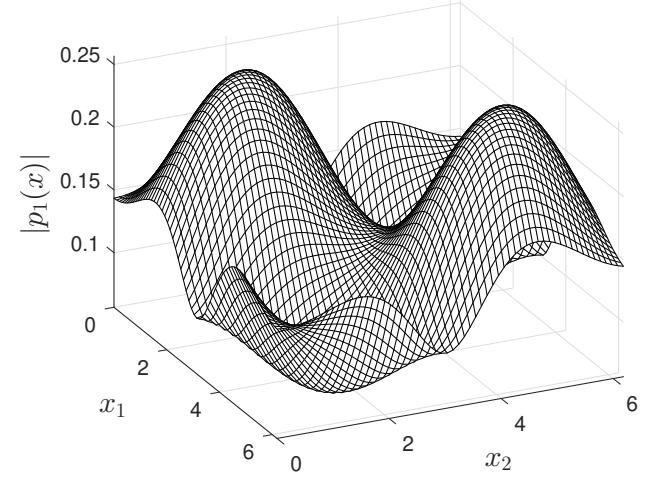

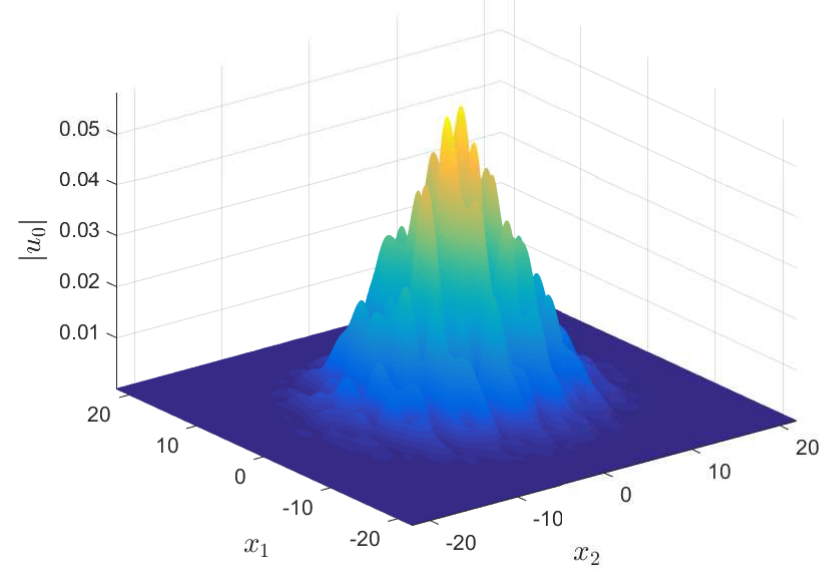

Figure 2. (a) The modulus of $p_{1}:=p_{n_{1}}\left(\cdot, k^{(1)}\right)$, where $n_{1}=4, k^{(1)}=$ $(-0.2,-0.4)$, see Fig. (1). (b) The modulus of the approximate ansatz $u^{\text {app }}(\cdot, 0)$ for $N=4$, the points $k^{(j)}, j=1, \ldots, 4$ as in Fig. (1), $\varepsilon=0.12$, and $A_{1}(X, 0)=\cdots=A_{4}(X, 0)=e^{-|X|^{2}}$.

Ansatz (1.2) for an approximate waves-packet uses $N$ elementary Bloch waves as carriers. Due to the slow dependence on $x$ of the envelope $A_{j}(\varepsilon x, \varepsilon t)$ the ansatz is concentrated in the Bloch 
variable $k$ near the points $k=k^{(1)}, \ldots, k^{(N)}$, see Sec. 6 for the definition of Bloch variables. In the formal derivation of the CMEs (1.3) we plug (1.2) in (1.1) and collect terms of (formally) equal orders of $\varepsilon$. All $O\left(\varepsilon^{1 / 2}\right)$ terms vanish due to the fact that $\left(\omega_{0}, p_{j}\right)$ are eigenpairs of (1.7) at $k=k^{(j)}, j=1, \ldots, N$. At $O\left(\varepsilon^{3 / 2}\right)$ both the nonlinearity and the term $\varepsilon W u^{\text {app }}$ contribute. We consider in the formal part only terms proportional to $e^{\mathrm{i} k^{(j)} \cdot x}, j=1, \ldots, N$ times a $2 \pi \mathbb{Z}^{d_{-}}$ periodic function, i.e. only terms concentrated in the Bloch variable near $k^{(j)}, j=1 \ldots, N$. For each $j \in\{1, \ldots, N\}$ we get

$$
\begin{aligned}
& \sum_{\substack{r \in\{1, \ldots, N\} \\
k^{(r)}=k^{(j)}}}\left(\mathrm{i}\left(\partial_{T} A_{r}+v_{g}^{(r)} \cdot \nabla A_{r}\right) p_{r}(x) e^{\mathrm{i} k^{(j)} \cdot x}\right)-\sum_{r=1}^{N} A_{r} \sum_{\substack{m \in\left\{-m_{*}, \ldots, m_{*}\right\} \\
k^{(r)}+l^{(m)} \in k^{(j)}+\mathbb{Z}^{d}}} a_{m} e^{\mathrm{i}\left(k^{(r)}+l^{(m)}\right) \cdot x} p_{r}(x) \\
& -\sigma(x) \sum_{\substack{(\alpha, \beta, \gamma) \in\{1, \ldots, N\}^{3} \\
k^{(\alpha)}-k^{(\beta)}+k(\gamma) \in k^{(j)}+\mathbb{Z}^{d}}} A_{\alpha} \bar{A}_{\beta} A_{\gamma} p_{\alpha}(x) \overline{p_{\beta}}(x) p_{\gamma}(x) e^{\mathrm{i}\left(k^{(\alpha)}-k^{(\beta)}+k^{(\gamma)}\right) \cdot x} .
\end{aligned}
$$

A necessary condition for the smallness of the overall residual for $u^{\text {app }}$ is that the $L^{2}(\mathbb{T})$ projection of these terms onto $p_{j}(\cdot) e^{\mathrm{i} k^{(j)}}$ vanishes. Because of the orthogonality of $\left\{p_{r}: r=\right.$ $\left.1, \ldots, N, k^{(r)}=k^{(j)}\right\}$ (see $(\mathrm{A} 1)$ ), this condition is exactly equation 1.3 ).

Of course, ansatz (1.2) with $\vec{A}$ satisfying (1.3) does not yield a zero residual in (1.1) because we omitted in $W u^{\text {app }}$ and in $|u|^{2} u$ terms not proportional to $e^{\mathrm{i} k^{(j)} \cdot x}, j=1, \ldots, N$ times a $2 \pi \mathbb{Z}^{d_{-}}$ periodic function and because only the projection of $(1.9)$ onto $p_{j}(\cdot) e^{\mathrm{i} k^{(j)}}$ was forced to vanish. In Sec. 6 we prove that, despite this, on time intervals of size $O\left(\varepsilon^{-1}\right)$ the residual is $O\left(\varepsilon^{5 / 2}\right)$ and the error between $u$ and $u^{\text {app }}$ is $O\left(\varepsilon^{3 / 2}\right)$.

\section{Asymptotic Construction of Gap Solitons of CMEs}

As explained in the introduction, one of the motivations for studying CMEs is the possible existence of gap solitons. When inserted into $u^{\text {app }}$ gap solitons generate nearly solitary wavepackets of 1.1 . Standing solitary waves of CMEs are of the form $\vec{A}(X, T)=e^{-\mathrm{i} \Omega T} \vec{B}(X)$, where $\vec{B}$ is spatially localized and satisfies

$$
\Omega \vec{B}=L_{\mathrm{CME}}(\nabla) \vec{B}-\vec{N}(\vec{B})
$$

where

$$
L_{\mathrm{CME}}(\nabla):=\left(\begin{array}{lll}
-\mathrm{i} v_{g}^{(1)} \cdot \nabla & & \\
& \ddots & \\
& & -\mathrm{i} v_{g}^{(N)} \cdot \nabla
\end{array}\right)-\kappa .
$$

For $\Omega \notin \operatorname{spec}\left(L_{\mathrm{CME}}\right)$ but asymptotically near a spectral edge an approximation via nonlinear Schrödinger (NLS) asymptotics is possible. These asymptotics require that the dispersion relation is locally (near the spectral edge) of a parabolic shape. Next, we provide a formal derivation of the asymptotic expansion in order to use this in a numerical construction of a standing gap soliton $\vec{A}(X, T)=e^{-\mathrm{i} \Omega T} \vec{B}(X)$.

The spectrum $\operatorname{spec}\left(L_{\mathrm{CME}}\right)$ is given by the range of $\Omega: \mathbb{R}^{d} \rightarrow \mathbb{R}$ as a solution of the dispersion relation, i.e. of

$$
\Omega(K) \in \operatorname{spec}\left(L_{\mathrm{CME}}(\mathrm{i} K)\right), K \in \mathbb{R}^{d} .
$$

Let us denote the $N$ eigenpairs of

$$
\Omega(K) \vec{\eta}(K)=L_{\mathrm{CME}}(\mathrm{i} K) \vec{\eta}(K)
$$

by $\left(\Omega_{j}(K), \vec{\eta}^{(j)}(K)\right), j=1, \ldots, N$ with $\Omega_{j}: \mathbb{R}^{d} \rightarrow \mathbb{R}, \vec{\eta}^{(j)}: \mathbb{R}^{d} \rightarrow \mathbb{R}^{N},\left\|\vec{\eta}^{(j)}(K)\right\|_{l^{2}\left(\mathbb{R}^{N}\right)}=1$. We make the following assumptions 
$\left(\mathrm{A} 1_{\mathrm{CME}}\right) \Omega_{*}:=\Omega_{j_{0}}\left(K_{0}\right) \in \partial\left(\operatorname{spec}\left(L_{\mathrm{CME}}\right)\right)$ for some $j_{0} \in\{1, \ldots, N\}, K_{0} \in \mathbb{R}^{d}$,

$\left(\mathrm{A} 2_{\mathrm{CME}}\right) \Omega_{j_{0}}(K)$ is a simple eigenvalue for all $K$ in a neighborhood of $K_{0}$,

$\left(\mathrm{A} 3_{\mathrm{CME}}\right)$ the Hessian $D^{2} \Omega_{j_{0}}\left(K_{0}\right)$ is definite,

The vanishing of $\nabla \Omega_{j_{0}}\left(K_{0}\right)$ is automatic as the edge is an extremal value of $\Omega_{j_{0}}$. Note that $\left(\mathrm{A} 3_{\mathrm{CME}}\right)$ implies that the extremum of $\Omega_{j_{0}}(K)$ at $K=K_{0}$ is isolated and (A2 $\left.2_{\mathrm{CME}}\right)$ impies that $K \mapsto \Omega_{j_{0}}(K)$ is analytic at $K_{0}$.

Our asymptotic ansatz for a gap soliton at $\Omega=\Omega_{*}+\varepsilon^{2} \lambda, 0<\varepsilon \ll 1$, is a slowly varying envelope $\varepsilon C(\varepsilon X)$ modulating the plane wave $e^{\mathrm{i} K_{0} \cdot X} \vec{\eta}^{\left(j_{0}\right)}\left(K_{0}\right)$. We define the ansatz in Fourier variables as:

$$
\Omega=\Omega_{*}+\varepsilon^{2} \lambda, \quad \overrightarrow{\hat{B}}(K) \sim \overrightarrow{\hat{B}}_{\text {ans }}(K):=\varepsilon^{1-d} \hat{C}\left(\frac{K-K_{0}}{\varepsilon}\right) \vec{\eta}^{\left(j_{0}\right)}(K),
$$

where the scalar $\lambda=O(1)$ and the envelope $C: \mathbb{R}^{d} \rightarrow \mathbb{R}$ are to be determined. Inserting $\Omega$ and $\vec{B}_{\text {ans }}$ into 2.1 , we obtain the residual

$\varepsilon^{1-d}\left[\Omega_{*}+\varepsilon^{2} \lambda-\Omega_{j_{0}}\left(K_{0}+\varepsilon \kappa\right)\right] \vec{\eta}^{\left(j_{0}\right)}\left(K_{0}+\varepsilon \kappa\right) \hat{C}(\kappa)+\beta(\kappa) \vec{\eta}^{(j)}\left(K_{0}+\varepsilon \kappa\right)+\widehat{\vec{N}}_{\perp}\left(\vec{B}_{\text {ans }}\right)\left(K_{0}+\varepsilon \kappa\right)$,

where

$$
\kappa:=\frac{K-K_{0}}{\varepsilon}, \quad \beta(\kappa):=\vec{\eta}^{\left(j_{0}\right)}\left(K_{0}+\varepsilon \kappa\right)^{T} \widehat{\vec{N}}\left(\vec{B}_{\text {ans }}\right)\left(K_{0}+\varepsilon \kappa\right)
$$

is the coefficient of the nonlinearity component parallel to $\vec{\eta}^{\left(j_{0}\right)}(K)$, and

$$
\widehat{\vec{N}}_{\perp}\left(\vec{B}_{\text {ans }}\right)\left(K_{0}+\varepsilon \kappa\right):=\widehat{\vec{N}}\left(\vec{B}_{\text {ans }}\right)\left(K_{0}+\varepsilon \kappa\right)-\beta(\kappa) \vec{\eta}^{(j 0)}\left(K_{0}+\varepsilon \kappa\right)
$$

is the orthogonal part. We expand

$$
\Omega_{j_{0}}\left(K_{0}+\varepsilon \kappa\right)=\Omega_{*}+\frac{1}{2} \varepsilon^{2} \kappa^{T} D^{2} \Omega_{j_{0}}\left(K_{0}\right) \kappa+O\left(\varepsilon^{3}\right) .
$$

The $O\left(\varepsilon^{1-d}\right)$ part of the residual then vanishes. Note that both $\beta$ and $\widehat{\vec{N}}_{\perp}\left(\vec{B}_{\text {ans }}\right)$ are $O\left(\varepsilon^{3-d}\right)$.

In order to make the $O\left(\varepsilon^{3-d}\right)$ part of the residual component parallel to $\eta^{\left(j_{0}\right)}(K)$ vanish, we set

$$
\lambda \hat{C}(\kappa)-\frac{1}{2} \kappa^{T} D^{2} \Omega_{j_{0}}\left(K_{0}\right) \kappa \hat{C}(\kappa)+\beta(\kappa)=0, \kappa \in \mathbb{R}^{d} .
$$

This effective equation for $C$ is not very practical due to the complicated structure of $\beta$. However, if we replace in $\beta$ the vector $\vec{\eta}^{\left(j_{0}\right)}\left(K_{0}+\varepsilon \kappa\right)$ by $\vec{\eta}^{\left(j_{0}\right)}\left(K_{0}\right)$, we formally arrive at $\beta(\kappa)=$ $\vec{\eta}^{\left(j_{0}\right)}\left(K_{0}\right)^{T} \vec{N}\left(\vec{\eta}^{\left(j_{0}\right)}\left(K_{0}\right)\right)(\hat{C} * \hat{C} * \hat{\bar{C}})(\kappa)$. The resulting effective equation in physical variables is the generalized NLS

$$
\lambda C+\frac{1}{2} \nabla^{T} D^{2} \Omega_{j_{0}}\left(K_{0}\right) \nabla C+\Gamma|C|^{2} C=0,
$$

where $\Gamma:=\vec{\eta}^{\left(j_{0}\right)}\left(K_{0}\right)^{T} \vec{N}\left(\vec{\eta}^{\left(j_{0}\right)}\left(K_{0}\right)\right)$. The above replacement can be shown to generate only higher order terms in the residual.

The orthogonal component $\widehat{\vec{N}}_{\perp}\left(\vec{B}_{\text {ans }}\right)$ of the $O\left(\varepsilon^{3-d}\right)$ part of the residual can be eliminated by adding to the ansatz $\overrightarrow{\hat{B}}_{\text {ans }}$ the correction term $\varepsilon^{3-d}\left(Q\left(L_{\mathrm{CME}}-\Omega_{*}\right) Q\right)^{-1} \widehat{\vec{N}}_{\perp}\left(\vec{B}_{\text {ans }}\right)$ with $Q:=$ $\chi_{B_{\varepsilon 1 / 2}\left(K_{0}\right)}(K)(I-P), P v:=\left(\eta^{\left(j_{0}\right)}(K)^{T} v\right) \eta^{\left(j_{0}\right)}(K)$. The invertibility of $Q\left(L_{\mathrm{CME}}-\Omega_{*}\right) Q$ follows for $\varepsilon>0$ small enough from assumption (A2 $\mathrm{CME}$ ). We do not employ this extended ansatz here as the leading order asymptotics suffice as an initial guess for our numerical computation of the solution $\vec{B}$ of $(2.1)$.

Based on the above asymptotics one expects that under assumptions $\left(\mathrm{A} 1_{\mathrm{CME}}\right)-\left(\mathrm{A} 3_{\mathrm{CME}}\right)$ the ansatz $\vec{B}_{\text {ans }}$ with a localized solution $C: \mathbb{R}^{d} \rightarrow \mathbb{C}$ of $(2.3)$ provides an approximation of a localized solution of (2.1). This can be made rigorous using a Lyapunov-Schmidt reduction 
argument. we refrain from this analysis here. In the following section we inspect whether assumptions (A $\left.1_{\mathrm{CME}}\right)-\left(\mathrm{A} 3_{\mathrm{CME}}\right)$ can be satisfied for $N=2,3,4$.

\section{CMEs for $N$ Linearly Resonant Modes with $N=2,3,4$}

We consider the perturbed PNLS (1.1) with $W$ in (A4). We study the dispersion relation $K \mapsto \Omega(K)$ of the resulting CMEs, where $K \in \mathbb{R}^{d}$ is the dual variable to $X$ with respect to the Fourier transform. A gap in the range of $\Omega$ is then a spectral gap of the corresponding spatial operator, i.e. of $L_{\mathrm{CME}}(\nabla)$. We concentrate on the existence of a spectral gap as one can typically expect the existence of standing solitary waves $\vec{A}(X, T)=e^{-\mathrm{i} \Omega T} \vec{B}(X)$ with an (exponentially) localized $\vec{B}$, a solution of $\Omega \vec{B}-L_{\mathrm{CME}}(\nabla) \vec{B}+\vec{N}(\vec{B})=0$, for $\Omega \notin \operatorname{spec}\left(L_{\mathrm{CME}}(\nabla)\right.$ ).

3.1. Two modes: $N=2$. Let us first choose $N=2$ in $(1.2)$. The CMEs $(1.3)$ have the form (3.1)

$$
\begin{aligned}
& \mathrm{i}\left(\partial_{T}+v_{g}^{(1)} \cdot \nabla\right) A_{1}+\kappa_{11} A_{1}+\kappa_{12} A_{2}+\left(\gamma_{1}^{(1,1,1)}\left|A_{1}\right|^{2}+2 \gamma_{1}^{(2,2,1)}\left|A_{2}\right|^{2}\right) A_{1}+\delta \gamma_{1}^{(2,1,2)} A_{2}^{2} \bar{A}_{1}=0 \\
& \mathrm{i}\left(\partial_{T}+v_{g}^{(2)} \cdot \nabla\right) A_{2}+\kappa_{22} A_{2}+\overline{\kappa_{12}} A_{1}+\left(\gamma_{2}^{(2,2,2)}\left|A_{2}\right|^{2}+2 \gamma_{2}^{(1,1,2)}\left|A_{1}\right|^{2}\right) A_{2}+\delta \gamma_{2}^{(1,2,1)} A_{1}^{2} \bar{A}_{2}=0,
\end{aligned}
$$

where

$$
\delta:=\left\{\begin{array}{l}
0 \text { if } k^{(1)}-k^{(2)} \notin \frac{1}{2} \mathbb{Z}^{d} \\
1 \text { if } k^{(1)}-k^{(2)} \in \frac{1}{2} \mathbb{Z}^{d} .
\end{array}\right.
$$

This is because only for $k^{(1)}-k^{(2)} \in \frac{1}{2} \mathbb{Z}^{d}$ is $2 k^{(2)}-k^{(1)} \in k^{(1)}+\mathbb{Z}^{d}$ such that the term $A_{2}^{2} \overline{A_{1}}$ appears in the first equation. Similarly $A_{1}^{2} \overline{A_{2}}$ appears in the second equation only then.

An example of a simple $W$ leading generically to $\kappa_{11}, \kappa_{22}, \kappa_{12} \neq 0$ is $W(x)=2 \cos \left(\left(k^{(1)}-\right.\right.$ $\left.\left.k^{(2)}\right) \cdot x\right)+1$. In that case is

$$
\begin{aligned}
& \kappa_{j j}=-1-\delta \int_{\mathbb{T}}\left|p_{j}(x)\right|^{2} \cos \left(2\left(k^{(2)}-k^{(1)}\right) \cdot x\right) \mathrm{d} x, j=1,2 \\
& \kappa_{12}=-\int_{\mathbb{T}} p_{2}(x) \overline{p_{1}}(x) \mathrm{d} x-\delta \int_{\mathbb{T}} p_{2}(x) \overline{p_{1}}(x) e^{2 \mathrm{i}\left(k^{(2)}-k^{(1)}\right) \cdot x} \mathrm{~d} x .
\end{aligned}
$$

In general, the dispersion relation $K \mapsto \Omega(K)$ of $(3.1)$ is given by

$$
\operatorname{det}\left(\begin{array}{cc}
\Omega-v_{g}^{(1)} \cdot K+\kappa_{11} & \kappa_{12} \\
\overline{\kappa 12} & \Omega-v_{g}^{(2)} \cdot K+\kappa_{22}
\end{array}\right)=0 .
$$

In one dimension $d=1$ we have $v_{g}^{(2)}=-v_{g}^{(1)}$ and $p_{2}=\overline{p_{1}}$ due to the band structure symmetry $\omega(k)=\omega(-k)$ and the fact that at most two Bloch waves exist for each frequency $\omega_{0}$. Then $\kappa_{11}=\kappa_{22} \in \mathbb{R}$ and thus

$$
\Omega(K)=-\kappa_{11} \pm \sqrt{v_{g}^{(1)^{2}} K^{2}+\left|\kappa_{12}\right|^{2}}
$$

such that there is the spectral gap $\left(-\kappa_{11}-\left|\kappa_{12}\right|,-\kappa_{11}+\left|\kappa_{12}\right|\right)$. In the one dimensional case CMEs (3.1) with $v_{g}^{(2)}=-v_{g}^{(1)}, \kappa_{12} \in \mathbb{R}, \delta \gamma_{1}^{(2,1,2)}=\delta \gamma_{2}^{(1,2,1)}=0$, and $\gamma_{1}^{(1,1,1)}=\gamma_{1}^{(2,2,1)}=\gamma_{2}^{(2,2,2)}=\gamma_{2}^{(1,1,2)}$ are well known to have a family of explicitly known solitary waves parametrized by the velocity $v \in\left(-v_{g}^{(1)}, v_{g}^{(1)}\right)$ and the frequency $\Omega \in\left(-\kappa_{11}-\left|\kappa_{12}\right|,-\kappa_{11}+\left|\kappa_{12}\right|\right)$, see [2]. In the general case (with an spectral gap) solitary waves can be constructed numerically, see [5].

For $d \geqslant 2$ we show that a gap exists only if $v_{g}^{(1)}$ and $v_{g}^{(2)}$ are linearly dependent and point in opposite directions. The non-existence of a gap is equivalent to the solvability of $(3.2)$ in $K \in \mathbb{R}^{d}$ for all $\Omega \in \mathbb{R}$. Writing $K=\rho j$ with $\rho \in \mathbb{R}$ and $j \in \mathbb{R}^{d},|j|=1$, we pose this as a problem 
in $\rho$ for fixed $\Omega \in \mathbb{R}$ and $j \in \mathbb{R}^{d}$. For the non-existence of a gap there must be for each $\Omega \in \mathbb{R}$ at least one direction $j$ such that a real solution $\rho$ exists. Assuming $j \cdot v_{g}^{(1)} \neq 0, j \cdot v_{g}^{(2)} \neq 0$, we get

$$
\begin{gathered}
\rho=\frac{1}{2\left(j \cdot v_{g}^{(1)}\right)\left(j \cdot v_{g}^{(2)}\right)}\left[\Omega j \cdot\left(v_{g}^{(1)}+v_{g}^{(2)}\right)+\kappa_{11} j \cdot v_{g}^{(2)}+\kappa_{22} j \cdot v_{g}^{(1)}\right. \\
\left. \pm\left(\left(j \cdot v_{g}^{(1)}\left(\Omega+\kappa_{22}\right)-j \cdot v_{g}^{(2)}\left(\Omega+\kappa_{11}\right)\right)^{2}+4\left|\kappa_{12}\right|^{2} j \cdot v_{g}^{(1)} j \cdot v_{g}^{(2)}\right)^{1 / 2}\right] .
\end{gathered}
$$

We write $j \cdot v_{g}^{(l)}=\left|v_{g}^{(l)}\right| \cos \left(\theta_{l}\right), l=1,2$ with $\theta_{l} \in[-\pi / 2, \pi / 2]$. A real $\rho$ exists if and only if

$$
\left(\left|v_{g}^{(1)}\right| \cos \left(\theta_{1}\right)\left(\Omega+\kappa_{22}\right)-\left|v_{g}^{(2)}\right| \cos \left(\theta_{2}\right)\left(\Omega+\kappa_{11}\right)\right)^{2} \geqslant-4\left|\kappa_{12}\right|^{2}\left|v_{g}^{(1)}\right|\left|v_{g}^{(2)}\right| \cos \left(\theta_{1}\right) \cos \left(\theta_{2}\right) .
$$

There are two cases: $\cos \left(\theta_{1}\right)=-\cos \left(\theta_{2}\right)$ (i.e. $v_{g}^{(1)}=-\alpha v_{g}^{(2)}$ with $\left.\alpha>0\right)$ and $\cos \left(\theta_{1}\right) \neq-\cos \left(\theta_{2}\right)$. In the former case 3.4 simplifies to

$$
\left(\Omega+\frac{\kappa_{22}\left|v_{g}^{(1)}\right|+\kappa_{11}\left|v_{g}^{(2)}\right|}{\left|v_{g}^{(1)}\right|+\left|v_{g}^{(2)}\right|}\right)^{2} \geqslant 4\left|\kappa_{12}\right|^{2} \frac{\left|v_{g}^{(1)}\right|\left|v_{g}^{(2)}\right|}{\left(\left|v_{g}^{(1)}\right|+\left|v_{g}^{(2)}\right|\right)^{2}}
$$

Hence, there is the spectral gap

$$
\left(-\frac{\kappa_{22}\left|v_{g}^{(1)}\right|+\kappa_{11}\left|v_{g}^{(2)}\right|}{\left|v_{g}^{(1)}\right|+\left|v_{g}^{(2)}\right|}-2\left|\kappa_{12}\right| \frac{\sqrt{\left|v_{g}^{(1)}\right|\left|v_{g}^{(2)}\right|}}{\left|v_{g}^{(1)}\right|+\left|v_{g}^{(2)}\right|},-\frac{\kappa_{22}\left|v_{g}^{(1)}\right|+\kappa_{11}\left|v_{g}^{(2)}\right|}{\left|v_{g}^{(1)}\right|+\left|v_{g}^{(2)}\right|}+2\left|\kappa_{12}\right| \frac{\sqrt{\left|v_{g}^{(1)}\right|\left|v_{g}^{(2)}\right|}}{\left|v_{g}^{(1)}\right|+\left|v_{g}^{(2)}\right|}\right) .
$$

Note that due to the continuous dependence of the solution $\rho$ on $j$, the excluded directions with $j \cdot v_{g}^{(1)}=0$ or $j \cdot v_{g}^{(2)}=0$ cannot change the existence of this gap.

In the latter case $\left(\cos \left(\theta_{1}\right) \neq-\cos \left(\theta_{2}\right)\right)$ it is either $v_{g}^{(1)}=\alpha v_{g}^{(2)}$ with $\alpha>0$ or $v_{g}^{(1)}$ and $v_{g}^{(2)}$ are linearly independent. If $v_{g}^{(1)}=\alpha v_{g}^{(2)}, \alpha>0$, then $\cos \left(\theta_{1}\right)=\cos \left(\theta_{2}\right)$ and the right hand side of (3.4) is non-positive, such that (3.4) always holds and there is no spectral gap. If $v_{g}^{(1)}$ and $v_{g}^{(2)}$ are linearly independent (i.e. $\left|\cos \left(\theta_{1}\right)\right| \neq\left|\cos \left(\theta_{2}\right)\right|$ ), then one can always choose $j$ such that $\cos \left(\theta_{1}\right)$ and $\cos \left(\theta_{2}\right)$ have equal signs leading to a non-positive right hand side of (3.4). Hence there is at least one direction in which (3.4) holds such that no spectral gap exists. Figure 3 (a) shows $\Omega=\Omega(K)$ for the case $d=2, \kappa_{11}=\kappa_{22}=0, \kappa_{12}=\kappa_{21}=1$, and $v_{g}^{(1)}=(0,1), v_{g}^{(2)}=(1,0)$.

The shape of the dispersion relation graph $\Omega=\Omega(K)$ near the edge of a spectral gap is important for the asymptotic construction of solutions in Sec. 2. Namely, the construction is based on a Taylor expansion of the band structure near an isolated localized extremum. For $N=2$ and $v_{g}^{(1)}=-\alpha v_{g}^{(2)}, \alpha>0$ there is no isolated extremum of $\Omega(K)$ at the spectral edges. This follows from (3.3), where the square root vanishes at both edges and the values of $\rho\left(\theta_{1}\right)$ for $\theta_{1} \in[0, \pi / 2)$ span a whole interval. From (3.3) one namely gets $\rho=\frac{1}{2 \alpha\left|v_{g}^{(2)}\right| \cos \left(\theta_{1}\right)}\left[\frac{\alpha-1}{\alpha+1}\left(\kappa_{22}+\alpha \kappa_{11} \mp 2 \sqrt{\alpha}\left|\kappa_{12}\right|\right)+\kappa_{11}-\alpha \kappa_{22}\right]$. This effectively one dimensional shape of the dispersion relation reflects the fact that with linearly dependent $v_{g}^{(1)}$ and $v_{g}^{(2)}$ the CMEs (2.1) both have the derivative in the same direction. Hence, the existence of solitary waves localized in all directions cannot be expected.

Figure 3 (b) shows $\Omega=\Omega(K)$ for the case $d=2, N=2, \kappa_{11}=\kappa_{22}=0, \kappa_{12}=\kappa_{21}=1$, and $v_{g}^{(1)}=-v_{g}^{(2)}=(1,1)$.

3.2. Odd number of modes: $N \in 2 \mathbb{N}+1$. First, we note that three (or more) linearly independent modes at one frequency $\omega_{0}$ are possible only for $d \geqslant 2$.

We interpret the dispersion relation $\operatorname{det}\left(L_{\mathrm{CME}}(\mathrm{i} K)-\Omega I_{N \times N}\right)=0$ as an algebraic equation in $\rho:=|K|$ for a given direction $K /|K| \in \mathbb{R}^{d}$ and a given $\Omega \in \mathbb{R}$. Because $L_{\mathrm{CME}}(\mathrm{i} K)$ is a Hermitian matrix for each $K$, the determinant of $L_{\mathrm{CME}}(\mathrm{i} K)-\Omega I_{N \times N}$ is real for each $K$. Hence, for odd 
(a)

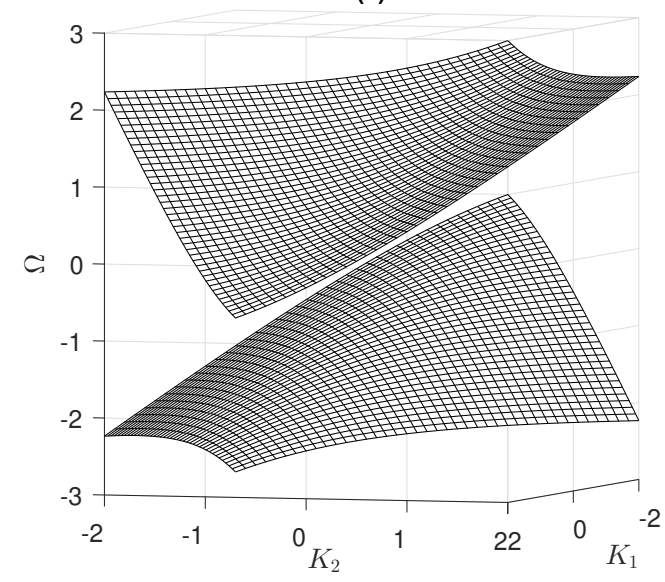

(b)

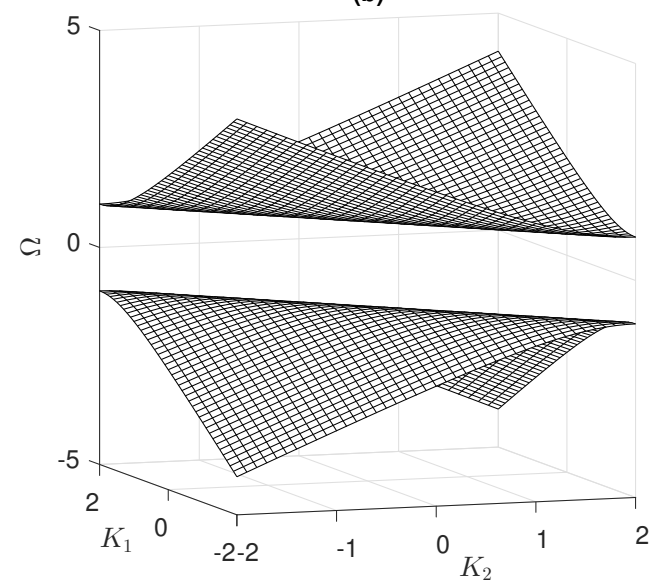

FiguRE 3. Dispersion relation of (3.1) with $\kappa_{11}=\kappa_{22}=0, \kappa_{12}=\kappa_{21}=1$. $v_{g}^{(1)}=(0,1), v_{g}^{(2)}=(1,0)$ in $(\mathrm{a})$ and $v_{g}^{(1)}=-v_{g}^{(2)}=(1,1)$ in $(\mathrm{b})$.

$N$ the dispersion relation is an algebraic equation of odd degree with real coefficients such that it has a real root for each $\Omega \in \mathbb{R}$. This implies the absence of spectral gaps for $N \in 2 \mathbb{N}+1$.

3.3. Four modes: $N=4$. We do not attempt to prove the existence of a gap for the general system (1.3) with $N=4$. Instead we show that in two dimensions $(d=2)$ under a simple condition on $\kappa$ a gap exists in the following symmetric case

$$
\begin{aligned}
& v_{g}^{(1)}=-v_{g}^{(2)}=: v, v_{g}^{(3)}=-v_{g}^{(4)}=: w, \\
& \kappa_{12}=\kappa_{34}=: \alpha_{1}, \\
& \kappa_{14}=\kappa_{32}=: \alpha_{2}, \\
& \kappa_{13}=\kappa_{42}=: \alpha_{3}, \\
& \kappa_{j j}=0, j=1, \ldots, 4,
\end{aligned}
$$

with $\alpha_{j} \in \mathbb{C}, j=1,2,3$. We also assume the linear independence of $v$ and $w$.

A simple example for which these symmetries hold is

$$
k^{(1)}=\left(l_{1}, l_{2}\right) \in \mathbb{B}, k^{(2)}=-k^{(1)}, k^{(3)}=\left(-l_{1}, l_{2}\right), k^{(4)}=-k^{(3)}
$$

with $l_{1} \neq 0, l_{2} \neq 0, n_{1}=\cdots=n_{4}=: n_{*}$, with a simple eigenvalue $\omega_{n_{*}}\left(k^{(j)}\right)$ for each $j=1, \ldots, 4$ , and with

$$
W(x)=a \cos \left(\left(k^{(2)}-k^{(1)}\right) \cdot x\right)+b \cos \left(\left(k^{(4)}-k^{(1)}\right) \cdot x\right)+c \cos \left(\left(k^{(3)}-k^{(1)}\right) \cdot x\right),
$$

$a, b, c \in \mathbb{R}$.

After the transformation

$$
(\xi, \eta)^{T}=\varphi\left(X_{1}, X_{2}\right):=\frac{1}{v_{1} w_{2}-w_{1} v_{2}}\left(\begin{array}{c}
w_{2} X_{1}-w_{1} X_{2} \\
v_{1} X_{2}-v_{2} X_{1}
\end{array}\right)
$$

system 1.3 becomes

$$
\begin{aligned}
& \mathrm{i}\left(\partial_{T}+\partial_{\xi}\right) A_{1}^{\prime}+\alpha_{1} A_{2}^{\prime}+\alpha_{3} A_{3}^{\prime}+\alpha_{2} A_{4}^{\prime}+N_{1}\left(\overrightarrow{A^{\prime}}\right)=0 \\
& \mathrm{i}\left(\partial_{T}-\partial_{\xi}\right) A_{2}^{\prime}+\overline{\alpha_{1}} A_{1}^{\prime}+\overline{\alpha_{2}} A_{3}^{\prime}+\overline{\alpha_{3}} A_{4}^{\prime}+N_{2}\left(\overrightarrow{A^{\prime}}\right)=0 \\
& \mathrm{i}\left(\partial_{T}+\partial_{\eta}\right) A_{3}^{\prime}+\overline{\alpha_{3}} A_{1}^{\prime}+\alpha_{2} A_{2}^{\prime}+\alpha_{1} A_{4}^{\prime}+N_{3}\left(\overrightarrow{A^{\prime}}\right)=0 \\
& \mathrm{i}\left(\partial_{T}-\partial_{\eta}\right) A_{4}^{\prime}+\overline{\alpha_{2}} A_{1}^{\prime}+\alpha_{3} A_{2}^{\prime}+\overline{\alpha_{1}} A_{3}^{\prime}+N_{4}\left(\overrightarrow{A^{\prime}}\right)=0,
\end{aligned}
$$


where $\vec{A}(\cdot, T)=\overrightarrow{A^{\prime}}(\varphi(\cdot), T)$. The dispersion relation of 3.6$)$ is given by

$$
\begin{aligned}
& \Omega^{4}-\Omega^{2}\left(K_{\xi}^{2}+K_{\eta}^{2}+2 \sum_{j=1}^{3}\left|\alpha_{j}\right|^{2}\right)+4 \Omega\left(\operatorname{Re}\left(\overline{\alpha_{1}} \alpha_{2} \alpha_{3}\right)+\operatorname{Re}\left(\alpha_{1} \overline{\alpha_{2}} \alpha_{3}\right)\right) \\
& +\left(K_{\xi} K_{\eta}+\left|\alpha_{2}\right|^{2}-\left|\alpha_{3}\right|^{2}\right)^{2}+\left|\alpha_{1}\right|^{2}\left(K_{\xi}^{2}+K_{\eta}^{2}+\left|\alpha_{1}\right|^{2}\right)=2 \operatorname{Re}\left(\left|\alpha_{1}\right|^{2} \alpha_{3}^{2}+\alpha_{2}^{2}{\overline{\alpha_{1}}}^{2}\right) .
\end{aligned}
$$

where $\left(K_{\xi}, K_{\eta}\right)$ is the dual variable to $(\xi, \eta)$. For $\Omega=0$ and $\left(K_{\xi}, K_{\eta}\right) \in \mathbb{R}^{2}$ the left hand side of this equation is larger than or equal to $\left|\alpha_{1}\right|^{4}$. The right hand side is smaller than or equal to $2\left|\alpha_{1}\right|^{2}\left(\left|\alpha_{2}\right|^{2}+\left|\alpha_{3}\right|^{2}\right)$. Hence, there is no real solution $\left(K_{\xi}, K_{\eta}\right) \in \mathbb{R}^{2}$ if $\left|\alpha_{1}\right|^{2}>2\left(\left|\alpha_{2}\right|^{2}+\left|\alpha_{3}\right|^{2}\right)$. As a result there is a spectral gap around 0 for $(3.6)$ if

$$
\left|\alpha_{1}\right|^{2}>2\left(\left|\alpha_{2}\right|^{2}+\left|\alpha_{3}\right|^{2}\right) \text {. }
$$

For a slightly less general case of the matrix $\kappa$ this gap was found already in [3]. Figure 4 shows an example of the dispersion relation of (1.3) with $N=4$ and (3.5) featuring a spectral gap. The plot in Fig 4 suggests that assumptions $\left(\mathrm{A} 1_{\mathrm{CME}}\right)-\left(\mathrm{A} 3_{\mathrm{CME}}\right)$ are satisfied at the lower edge

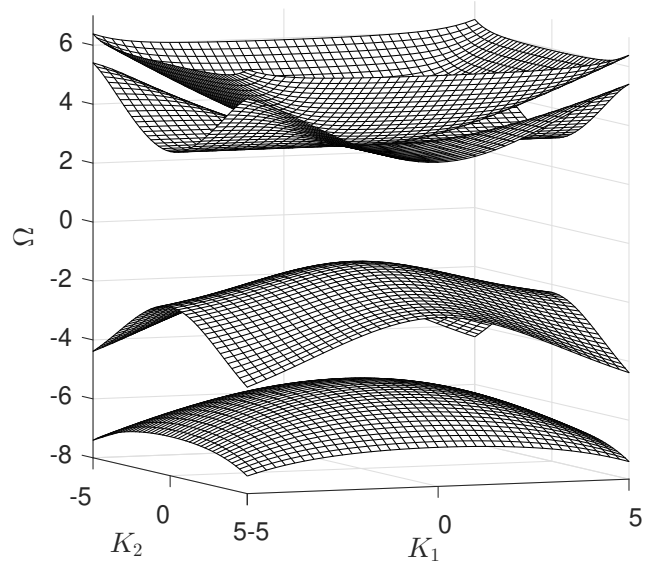

Figure 4. Dispersion relation of $(1.3)$ with $N=4, v_{g}^{(j)}$ and $\kappa$ given in (3.5), and $\alpha_{1}=3, \alpha_{2}=\alpha_{3}=1, v=(0,1), w=(1,0)$.

of the spectral gap with $K_{0}:=(0,0)^{T}$. In Sec. 4 we compute corresponding solitary waves.

\section{Numerical Example of Standing Solitary Waves of the CMEs}

In order to compute standing solitary waves $\vec{A}(X, t)=e^{-\mathrm{i} \Omega T} \vec{B}(X)$, we perform a fixed point iteration on (2.1) starting with the initial guess given by 2.2 with a localized solution $C$ of (2.3). The fixed point iteration of (2.1) is performed using the Petviashvili iteration [15, 1 ] in Fourier variables because standard finite difference or finite element discretizations of (2.1) lead to spurious checkerboard oscillations caused by the decoupling of even and odd nodes- an effect referred to as Fermion doubling in the physics literature, see e.g. Sec. 5 in [11].

To find a localized solution $C$ of $(2.3)$, we first compute a radially symmetric $C(y)=C_{\mathrm{rad}}(\rho)$ with $\rho:=|y|$ of 2.3 with $D^{2} \eta^{\left(j_{0}\right)}$ replaced by $\mu I$, e.g. with $\mu:=\frac{1}{d} \sum_{j=1}^{d} \mu_{j}$, where $\left\{\mu_{1}, \ldots, \mu_{d}\right\}$ are the eigenvalues of $D^{2} \Omega^{\left(j_{0}\right)}\left(K_{0}\right)$. We use the shooting method for the computation of $C_{\text {rad }}$. Then we perform a parameter continuation in order to deform $C_{\text {rad }}$ to a solution of (2.3). Hence, the algorithm is

1) shooting method for a solution $C_{\mathrm{rad}}$ of

$$
\lambda C_{\mathrm{rad}}+\mu\left(C_{\mathrm{rad}}^{\prime \prime}(\rho)+\frac{d-1}{\rho} C_{\mathrm{rad}}^{\prime}(\rho)\right)+\Gamma C_{\mathrm{rad}}^{3}(\rho)=0, \quad \rho \in(0, \infty),
$$


with $C_{\text {rad }}(\rho) \rightarrow 0$ as $\rho \rightarrow \infty$

2) parameter continuation in the parameter $\nu \in[0,1]$ (starting at $\nu=0$ and terminating at $\nu=1$ ) on equation (2.3) with $D^{2} \eta^{\left(j_{0}\right)}$ replaced by $\mu I+\nu\left(D^{2} \eta^{\left(j_{0}\right)}-\mu I\right)$; finite difference discretization in the $x$-variable,

3 ) evaluation of 2.2 with $C$ from the previous step at $\nu=1$ and with a chosen (small) $\varepsilon>0$,

4) Petviashvili iteration on (2.1) with $\Omega=\Omega_{*}+\varepsilon^{2} \alpha$ using the initial guess given by (2.2),

5 ) parameter continuation in the parameter $\Omega$ from $\Omega_{*}+\varepsilon^{2} \alpha$ to the desired value of $\Omega$ in the spectral gap of $L_{\mathrm{CME}}$ (Petviashvili iteration at each continuation step).

Example 1. We consider the two dimensional $(d=2)$ case with $N=4$ and with the symmetries (3.5). The parameters are chosen like in Figure 4, i.e. $\alpha_{1}=3, \alpha_{2}=\alpha_{3}=1, v=(0,1), w=$ $(1,0)$. The spectral gap is approximately $(-1,3)$. We let $\Omega_{*}$ be the lower edge, i.e. $\Omega_{*} \approx$ -1 . Using the notation of Sec. 2, it is $\Omega_{*}=\Omega_{j_{0}}\left(K_{0}\right)$ with $j_{0}=2, K_{0}=0, \eta^{\left(j_{0}\right)}\left(K_{0}\right)=$ $\frac{1}{2}(-1,-1,1,1)^{T}$. A numerical approximation via finite differences yields

$$
D^{2} \Omega_{j_{0}}\left(K_{0}\right) \approx-\frac{1}{4} I_{2 \times 2}, \Gamma \approx 2.125 .
$$

In Figure 5 (a) we plot the radial profile of the radially symmetric solution $C_{\text {rad }}$ of the NLS. Because $D^{2} \Omega_{j_{0}}\left(K_{0}\right)$ is a multiple of the identity, it is $C=C_{\text {rad }}$. Figure 5 (b) shows the first component of the asymptotic ansatz 2.2 with $\varepsilon=0.1$. Due to $K_{0}=0$ and the realness of $C$ it is $\vec{B}_{\text {ans }}: \mathbb{R}^{2} \rightarrow \mathbb{R}^{4}$. Moreover, due to the form of $\eta^{\left(j_{0}\right)}\left(K_{0}\right)$ we have $B_{\text {ans }, 1}=B_{\text {ans }, 2}=-B_{\text {ans }, 3}=$ $-B_{\text {ans }, 4}$. Figure 5 (c) shows the numerically computed (via the Petviashvili iteration) solution $\vec{B}$ at $\Omega=\Omega_{*}+\varepsilon^{2}=-0.99$. Only $B_{1}$ and $B_{3}$ are plotted since the computed solution satisfies $B_{2}=\overline{B_{1}}, B_{4}=\overline{B_{3}}$. In Figure 6 we plot the components $B_{1}$ and $B_{3}$ of the solution to (2.1) at $\Omega=-0.8$ obtained by a parameter continuation in $\Omega$ from $\Omega_{*}+\varepsilon^{2}$. During the continuation the symmetry $B_{1}=B_{2}=-B_{3}=-B_{4}$ seems to break. Also note that the solution at $\Omega=-0.8$ is far from radially symmetric.

\section{Moving Gap Solitons}

An important feature of the CMEs in one dimension $(d=1)$ is the existence of a whole family of solitary waves parametrized by their velocity. It is natural to ask whether this holds also for $d>1$. As explained in the introduction, the equal $\varepsilon$-scaling of $t$ and $x$ in the asymptotic ansatz for a waves-packet of the PNLS would (in the affirmative case) imply that nearly solitary waves of the PNLS exist with a whole range of velocities. However, as we show next, for $N \leqslant 4$ and the most natural ansatz there seem to be no moving solitary waves in spectral gaps.

The simplest ansatz for a moving solitary wave of the time dependent CMEs $(1.3)$ is $\vec{A}(X, T)=$ $e^{-\mathrm{i} \Omega^{(v)} T} \vec{B}^{(v)}(X-v T)$ with some $v \in \mathbb{R}^{d}, \Omega^{(v)} \in \mathbb{R}$, and some localized $\overrightarrow{B^{(v)}}: \mathbb{R}^{d} \rightarrow \mathbb{C}^{N}$ $\left(\left|\vec{B}^{(v)}(Y)\right| \rightarrow 0\right.$ as $\left.|Y| \rightarrow \infty\right)$. $\vec{B}^{(v)}$ must satisfy

$$
\Omega^{(v)} \vec{B}^{(v)}=\left(L_{\mathrm{CME}}(\nabla)+\mathrm{i} v \cdot \nabla\right) \vec{B}^{(v)}-\vec{N}\left(\vec{B}^{(v)}\right) .
$$

The corresponding dispersion relation is given by $K \mapsto \Omega^{(v)}(K)$, where

$$
\Omega_{j}^{(v)}(K)=\Omega_{j}^{(0)}(K)-v \cdot K, K \in \mathbb{R}^{d}, j \in\{1, \ldots, N\} .
$$

An exponentially localized $\vec{B}^{(v)}$ is expected in spectral gaps, i.e. for $\Omega^{(v)} \in \mathbb{R} \backslash \cup_{j=1}^{N} \Omega_{j}^{(v)}\left(\mathbb{R}^{d}\right)$. We have, however, the following

Lemma 1. Let $v \in \mathbb{R}^{d}$. If for some $j_{*} \in\{1, \ldots, N\}$ and some direction $\xi \in \mathbb{R}^{d}$, such that $\xi^{T} v \neq 0$, it is $\Omega_{j_{*}}^{(0)}(K) \rightarrow$ const. for $K=r \xi, \mathbb{R} \ni r \rightarrow \pm \infty$, then $\mathbb{R}=\Omega_{j_{*}}^{(v)}(\mathbb{R} \xi)$. Hence, in this case, there is no spectral gap of (5.1). 

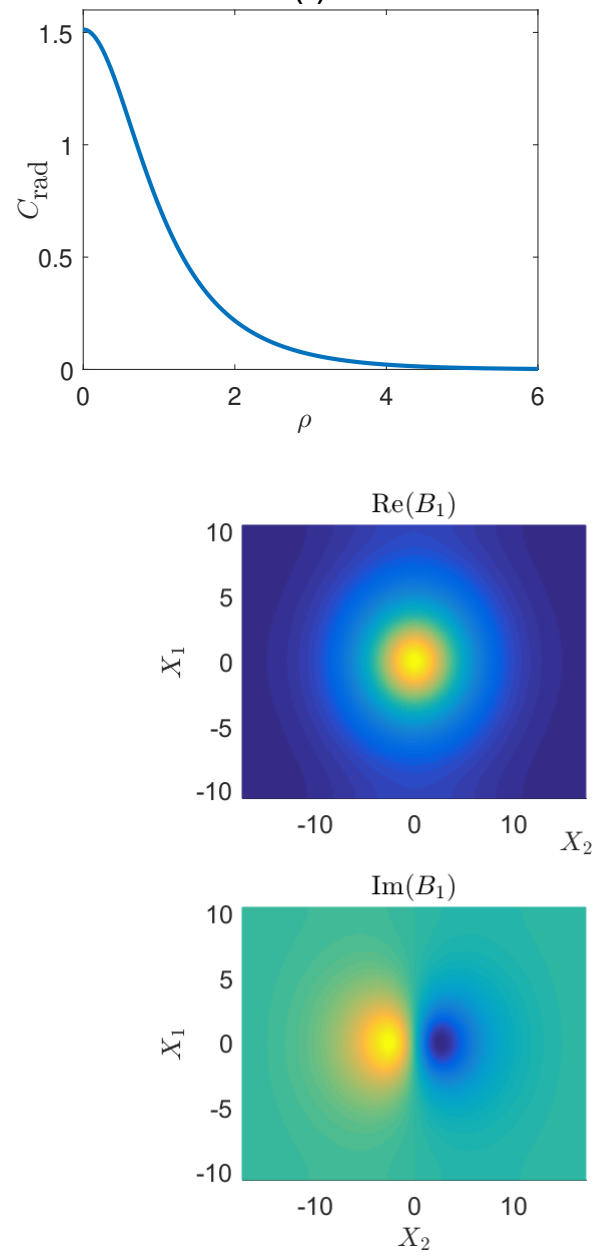

(b)

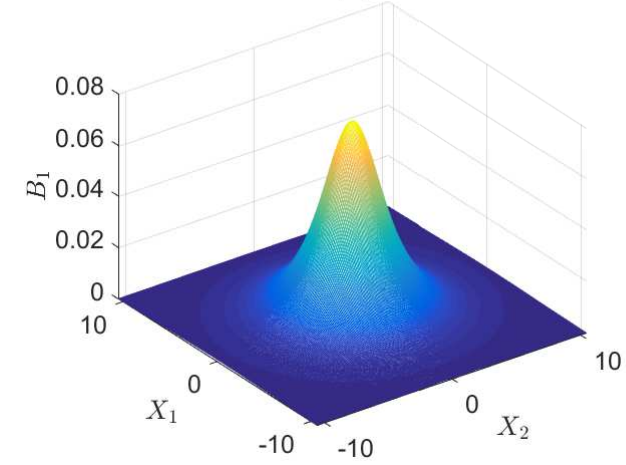

(c)
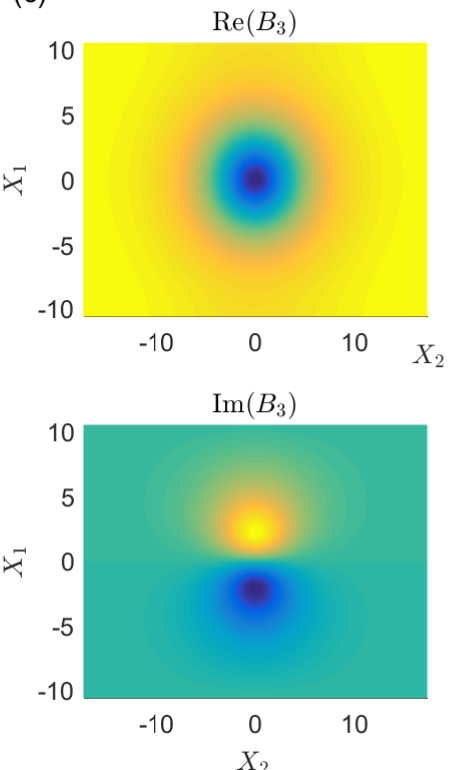

Figure 5. Plots for Example 1. (a) The profile of the positive solution $C_{\text {rad }}$ of 4.1 ; (b) $B_{\text {ans, } 1}$ at $\varepsilon=0.1$; (c) $B_{1}$ and $B_{3}$ at $\Omega=\Omega_{*}+\varepsilon^{2}=-0.99$.

Proof. The graph $K \mapsto v \cdot K$ is a hyperplane in $\mathbb{R}^{d+1}$. Therefore, if the eigenvalue $\Omega_{j_{*}}^{(0)}, j_{*} \in$ $\{1, \ldots, N\}$ is asymptotically horizontal along the direction $\xi \in \mathbb{R}^{d}$ not orthogonal to $v$, then $\Omega_{j_{*}}^{(v)}(K)=\Omega_{j}^{(0)}(K)-v \cdot K \rightarrow \infty$ for $K=r \xi, \mathbb{R} \ni r \rightarrow \infty$ or $r \rightarrow-\infty$. In the opposite direction $\Omega_{j_{*}}^{(v)}(K) \rightarrow-\infty$. Due to the intermediate value theorem it is $\Omega_{j_{*}}^{(v)}(\mathbb{R} \xi)=\mathbb{R}$.

A natural approach to constructing moving solitary waves is a parameter continuation from standing solitary waves (i.e. those with $v=0$ ). Based on the discussion in Sec. 3 we do not expect (exponentially) localized solitary waves for $N \leqslant 3$ and $d \geqslant 2$. For $N=4$ and $d=2$ Example 1 provides a setting with a spectral gap and standing solitary waves. Unfortunately, for $N=4$ and the symmetric case (3.5) (satisfied by Example 1) the above mentioned horizontal asymptotics of the dispersion relation graph are always satisfied:

Lemma 2. Let $N=4, d=2$ and let (3.5) be satisfied. Then for each $\gamma \in \mathbb{R} \backslash\left(-\left|\alpha_{1}\right|,\left|\alpha_{1}\right|\right)$ there exists a direction $\theta \in(-\pi, \pi]$ and $j \in\{1, \ldots, 4\}$ such that $\Omega_{j}((r \cos (\theta), r \sin (\theta))) \rightarrow \gamma$ as $r \rightarrow \infty$.

Proof. Writing $K_{\xi}=r \cos (\theta)$ and $K_{\eta}=r \sin (\theta)$ with $r>0, \theta \in(-\pi, \pi]$, the lemma is proved if the dispersion relation (3.7) has a solution $\theta \in(-\pi, \pi]$ for each $|\Omega| \geqslant\left|\alpha_{1}\right|$ and for all $r>0$ 

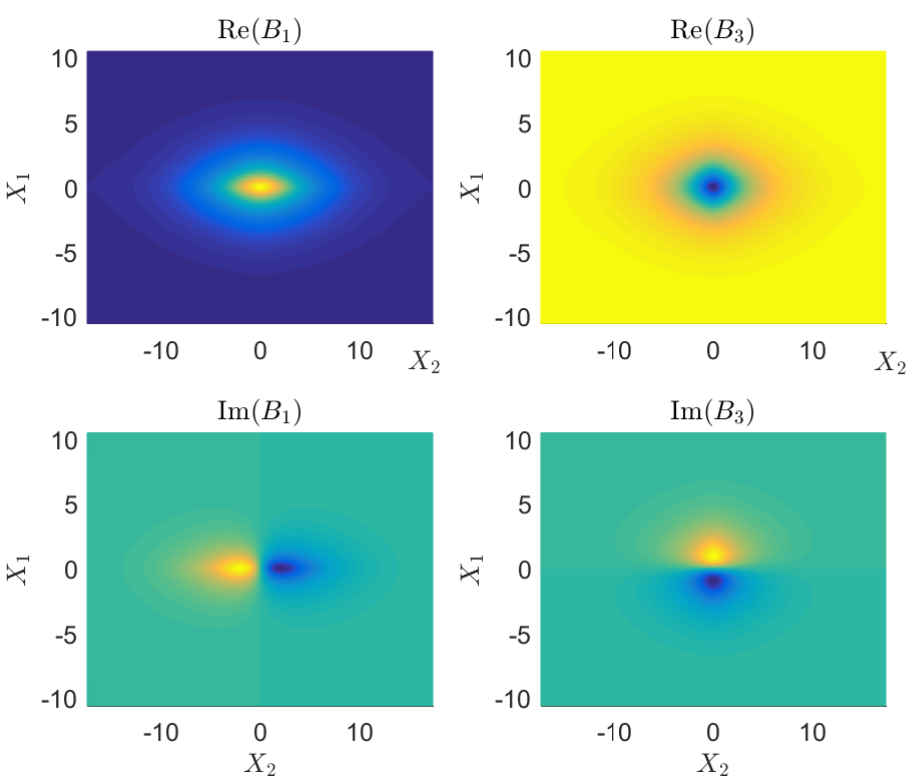

Figure 6. Plots for Example 1, $B_{1}$ and $B_{3}$ at $\Omega=-0.8$.

large enough. Dispersion relation 3.7 can be rewritten as

$$
\left(\frac{r^{2}}{2} \sin (2 \theta)+\left|\alpha_{2}\right|^{2}-\left|\alpha_{3}\right|^{2}\right)^{2}=r^{2}\left(\Omega^{2}-\left|\alpha_{1}\right|^{2}\right)-\psi
$$

where

$$
\psi:=\Omega^{4}-2 \Omega^{2} \sum_{j=1}^{3}\left|\alpha_{j}\right|^{2}+4 \Omega\left(\operatorname{Re}\left(\overline{\alpha_{1}} \alpha_{2} \alpha_{3}+\alpha_{1} \overline{\alpha_{2}} \alpha_{3}\right)\right)+\left|\alpha_{1}\right|^{4}-2\left|\alpha_{1}\right|^{2} \operatorname{Re}\left(\alpha_{3}^{2}\right)-2 \operatorname{Re}\left({\overline{\alpha_{1}}}^{2} \alpha_{2}^{2}\right) .
$$

Hence

$$
\sin (2 \theta)= \pm \frac{2}{r^{2}}\left(\left|\alpha_{3}\right|^{2}-\left|\alpha_{2}\right|^{2} \pm \sqrt{r^{2}\left(\Omega^{2}-\left|\alpha_{1}\right|^{2}\right)-\psi}\right) .
$$

For $r$ large a real solution $\theta$ clearly exists if and only if $|\Omega| \geqslant\left|\alpha_{1}\right|$.

Figure 4 shows that for Example 1 the eigenvalue $\Omega_{3}^{(0)}(K)$ converges to the constant $\alpha_{1}=$ 3 (the upper gap edge) along two lines in $\mathbb{R}^{2}$. In Figure 7 we plot the dispersion relation $\left(K, \Omega^{(v)}(K)\right)$ for the velocity $v=(1,1)^{T}$. Clearly, no gap occurs.

\section{Justification of CMEs (1.3) as Amplitude Equations}

In this section we prove Theorem 1, This provides a rigorous justification of the CMEs (1.3) as amplitude equations for waves-packets approximated by the formal ansatz (1.2).

In theorem 1 the functions $\hat{A}_{j}$ are Fourier transformations of $A_{j}$, where

$$
\hat{f}(k):=\frac{1}{(2 \pi)^{d}} \int_{\mathbb{R}^{d}} f(x) e^{-\mathrm{i} k \cdot x} \mathrm{~d} x
$$

with the inverse $f(x)=\int_{\mathbb{R}^{d}} \hat{f}(k) e^{\mathrm{i} k \cdot x} \mathrm{~d} k$. The space $L_{r}^{1}\left(\mathbb{R}^{d}\right)$ is

$$
L_{r}^{1}\left(\mathbb{R}^{d}\right):=\left\{f \in L^{1}\left(\mathbb{R}^{d}\right):\|f\|_{L_{r}^{1}\left(\mathbb{R}^{d}\right)}:=\int_{\mathbb{R}^{d}}(1+|x|)^{r}|f(x)| \mathrm{d} x<\infty\right\} .
$$

We first summarize the functional analytic tools and some well known results regarding these. A more detailed presentation can be found, e.g., in [7]. 


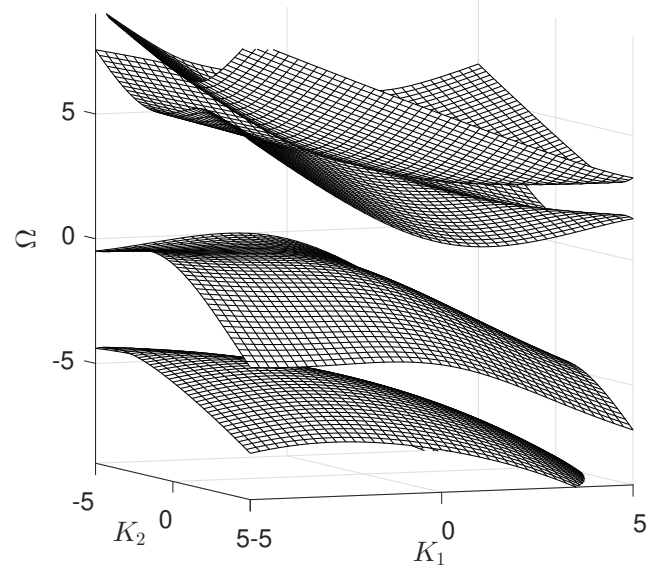

Figure 7. Dispersion relation for (5.1) with $d=2, N=4, v=(0.3,0.3)^{T}$ and $v_{g}^{(j)}, j=1, \ldots, 4$, and $\kappa$ like in Example 1 .

We use the Bloch transformation

$$
\begin{aligned}
& \mathcal{T}: \quad f \mapsto \mathcal{T}(f):=\tilde{f}, \\
& \tilde{f}(x, k):=\sum_{\eta \in \mathbb{Z}^{d}} \widehat{f}(k+\eta) e^{\mathrm{i} \eta \cdot x},
\end{aligned}
$$

which is an isomorphism from $H^{s}\left(\mathbb{R}^{d}\right)$ to $L^{2}\left(\mathbb{B}, H^{s}(\mathbb{T})\right)$ for $s \geqslant 0$ with the inverse

$$
f(x)=\int_{\mathbb{B}} \tilde{f}(x, k) e^{\mathrm{i} k \cdot x} \mathrm{~d} k .
$$

The following properties hold for all $f, g \in H^{s}\left(\mathbb{R}^{d}\right)$ and $1 \leqslant j \leqslant d, \mathbb{N} \ni p \leqslant s$ :

$$
\begin{gathered}
\mathcal{T}(f)\left(x, k+e_{j}\right)=e^{-\mathrm{i} x_{j}} \mathcal{T}(f)(x, k), \\
\mathcal{T}\left(\partial_{x_{j}}^{p} f\right)(x, k)=\left(\partial_{x_{j}}+\mathrm{i} k_{j}\right)^{p}(\mathcal{T} f)(x, k), \\
\mathcal{T}(V f)(x, k)=V(x) \mathcal{T}(f)(x, k), \text { if } V \in C(\mathbb{T}, \mathbb{C}), \\
\mathcal{T}(f g)(x, k)=((\mathcal{T} f) * \mathbb{B}(\mathcal{T} g))(x, k):=\int_{\mathbb{B}}(\mathcal{T} f)(x, k-l)(\mathcal{T} g)(x, l) \mathrm{d} l .
\end{gathered}
$$

It is, however, inconvenient to carry out the estimates of the residual and of the asymptotic error in the $H^{s}\left(\mathbb{R}^{d}\right)$-norm (or equivalently in the $L^{2}\left(\mathbb{B}, H^{s}(\mathbb{T})\right.$ )-norm in the Bloch variables). The reason is the loss of $\varepsilon$-powers when evaluating the $L^{2}$-norm if a function of the form $f(\varepsilon \cdot)$. We prove Theorem 1 by working in an $L^{1}$ space in the Bloch variables. Estimate (1.5) is then obtained by an analog of the Lebesgue-Riemann lemma. Namely, if $\tilde{f} \in L^{1}\left(\mathbb{B}, H^{s}(\mathbb{T})\right)$ with $s>d / 2$ and such that (6.3) holds, then the function $f(x):=\int_{\mathbb{B}} \tilde{f}(x, k) e^{\mathrm{i} k \cdot x} \mathrm{~d} k$ satisfies

$$
\sup _{x \in \mathbb{R}^{d}}|f(x)| \leqslant c\|\tilde{f}\|_{L^{1}\left(\mathbb{B}, H^{s}(\mathbb{T})\right)} \quad \text { and } u(x) \rightarrow 0 \text { as }|x| \rightarrow \infty .
$$

See Lemma 1 in [7]. This $L^{1}$ approach is one of the major differences from [9], where the authors work in the space $H_{\varepsilon}^{s}$, see Sec. 1. Note that our assumption (A3) corresponds to the assumption of a "closed mode system of order 3" in 9. There, however, the error is estimated by $C \varepsilon$ in the $H_{\varepsilon}^{s}$-norm, which produces the estimate $C \varepsilon^{1-d / 2}$ in the $L^{\infty}\left(\mathbb{R}^{d}\right)$-norm. For $C \varepsilon^{3 / 2}$, like in Theorem 1, the assumption of a closed mode system of a higher order (i.e. a stricter 
assumption than (A3)) is needed in [9]. As mentioned above, our approach also prevents us from having to consider high order correction terms in the asymptotic expansion of the solution.

Next, because the eigenfunctions $\left(p_{n}(\cdot, k)\right)_{n \in \mathbb{N}}$ are complete in $L^{2}(\mathbb{T})$, we define the expansion/diagonalization operator

$$
\mathcal{D}: L^{1}\left(\mathbb{B}, H^{s}(\mathbb{T})\right) \rightarrow \mathcal{X}(s):=L^{1}\left(\mathbb{B}, l_{s / d}^{2}\right), \quad \widetilde{u} \mapsto \vec{U}:=\left(U_{n}\right)_{n \in \mathbb{N}},
$$

where $\tilde{u}(x, k)=\sum_{n \in \mathbb{N}} U_{n}(k) p_{n}(x, k), U_{n}(k)=\left\langle\widetilde{u}(\cdot, k, t), p_{n}(\cdot, k)\right\rangle_{L^{2}(\mathbb{T})} . \mathcal{D}$ is an isomorphism, see Lemma 6 in [7]. Note that $\|\vec{U}\|_{\mathcal{X}(s)}:=\int_{\mathbb{B}}\|\vec{U}(k)\|_{l_{s / d}^{2}} \mathrm{~d} k$ and

$$
l_{s / d}^{2}:=\left\{\vec{v}=\left(v_{n}\right)_{n \in \mathbb{N}} \in l^{2}\left(\mathbb{R}^{d}\right):\|\vec{v}\|_{l_{s / d}^{2}}^{2}=\sum_{n \in \mathbb{N}} n^{\frac{2 s}{d}}\left|v_{n}\right|^{2}<\infty\right\} .
$$

6.1. Choice of an extended ansatz. We return now to the formal asymptotic ansatz (1.2). Applying $\mathcal{T}$, we get

$$
\tilde{u}^{\mathrm{app}}(x, k, t)=\varepsilon^{1 / 2-d} \sum_{\eta \in \mathbb{Z}^{d}} \sum_{j=1}^{N} \hat{A}_{j}\left(\frac{k-k^{(j)}+\eta}{\varepsilon}, \varepsilon t\right) p_{j}(x) e^{-\mathrm{i} \omega_{0} t} e^{\mathrm{i} \eta \cdot x} .
$$

The Gross-Pitaevskii equation (1.1) transforms to

$$
\left(\mathrm{i} \partial_{t}-\mathcal{L}(x, k)\right) \tilde{u}(x, k, t)-\varepsilon \sum_{m=-m_{*}}^{m_{*}} a_{m} \tilde{u}\left(x, k-l^{(m)}, t\right)-\sigma(x)\left(\tilde{u} *_{\mathbb{B}} \tilde{\bar{u}} *_{\mathbb{B}} \tilde{u}\right)(x, k, t)=0 .
$$

Here we have used the fact that for $g(x):=f(x) e^{\mathrm{i} l \cdot x}$ is $(\mathcal{T} g)(x, k)=(\mathcal{T} f)(x, k-l)$.

The Bloch transformed residual of $u^{\text {app }}$ in the Gross-Pitaevskii (GP) equation is (6.11)

$$
\begin{aligned}
& \mathcal{T}\left(\operatorname{GP}\left(u^{\mathrm{app}}\right)\right)(x, k, t)=\varepsilon^{3 / 2-d} e^{-\mathrm{i} \omega_{0} t} \sum_{\eta \in \mathbb{Z}^{d}} e^{\mathrm{i} \eta \cdot x} \sum_{j=1}^{N}\left[\mathrm{i} \partial_{T} \hat{A}_{j}\left(\frac{k-k^{(j)}+\eta}{\varepsilon}, T\right) p_{j}(x)\right. \\
& +2 \mathrm{i} \hat{A}_{j}\left(\frac{k-k^{(j)}+\eta}{\varepsilon}, T\right) \frac{k-k^{(j)}+\eta}{\varepsilon} \cdot \nabla p_{j}(x)-\sum_{m=-m_{*}}^{m_{*}} a_{m} \hat{A}_{j}\left(\frac{k-k^{(j)}-l^{(m)}+\eta}{\varepsilon}, T\right) p_{j}(x) \\
& \left.-\varepsilon\left|\frac{k-k^{(j)}+\eta}{\varepsilon}\right|^{2} \hat{A}_{j}\left(\frac{k-k^{(j)}+\eta}{\varepsilon}, T\right) p_{j}(x)-I(x, k, t)\right],
\end{aligned}
$$

where

$$
\begin{aligned}
& I(x, k, t)=\sigma(x) \sum_{\substack{\alpha, \beta, \gamma \\
\eta^{(\alpha)}, \eta^{(\beta)}, \eta^{(\gamma)}}} p_{\alpha} \bar{p}_{\beta} p_{\gamma} e^{\mathrm{i}\left(\eta^{(\alpha)}-\eta^{(\beta)}+\eta^{(\gamma)}\right) \cdot x} \int_{\frac{\mathbb{B}+k(\beta)-k(\gamma)-\eta^{(\beta)}+\eta^{(\gamma)}}{\varepsilon}} \int_{\frac{\mathbb{B}-k(\gamma)+\eta^{(\gamma)}}{\varepsilon}} \\
& \hat{A}_{\alpha}\left(\frac{k-k^{(\alpha)}+k^{(\beta)}-k^{(\gamma)}+\eta^{(\alpha)}-\eta^{(\beta)}+\eta^{(\gamma)}}{\varepsilon}-\tilde{h}, T\right) \hat{\bar{A}}_{\beta}(\tilde{h}-\tilde{l}, T) \hat{A}_{\gamma}(\tilde{l}, T) d \tilde{l} d \tilde{h} .
\end{aligned}
$$

The $O\left(\varepsilon^{1 / 2-d}\right)$ terms in $\mathcal{T}\left(\operatorname{GP}\left(u^{\text {app }}\right)\right)$ vanish due to the fact that each $p_{j}$ is an eigenfunction of $\mathcal{L}\left(\cdot, k^{(j)}\right)$ with the same eigenvalue $\omega_{0}$. The nonlinearity term was calculated using straightforward variable transformations in the convolution integrals. 
Note that for $\hat{A}_{j}(K, T), j=1, \ldots, N$ concentrated near $K=0$ one can formally approximate $\hat{A}_{j}(K, T)$ by $\chi_{B_{\varepsilon-1 / 2}(0)}(K) \hat{A}_{j}(K, T)$. We show below in Sec. 6.2 that $I(x, \cdot, t)$ can then be approximated near $k=k^{(j)}$ (precisely on $B_{\varepsilon^{1 / 2}}\left(k^{(j)}\right)$ ) by

$$
\sum_{\substack{\alpha, \beta, \gamma \in\{1, \ldots, N\} \\ k^{(\alpha)}-k^{(\beta)}+k^{(\gamma)} \in k^{(j)}+\mathbb{Z}^{d}}}\left(\hat{A}_{\alpha} * \hat{\bar{A}}_{\beta} * \hat{A}_{\gamma}\right)\left(\frac{k-k^{(j)}}{\varepsilon}, T\right) e^{\mathrm{i}\left(k^{(\alpha)}-k^{(\beta)}+k^{(\gamma)}-k^{(j)}\right) \cdot x} p_{\alpha}(x) \bar{p}_{\beta}(x) p_{\gamma}(x) .
$$

The residual (6.11) is concentrated (in $k$ ) near the points $k^{(j)}, j=1, \ldots, N$ but also near other points in $\mathbb{B}$. These are, firstly, the concentration points of those terms in $I$, for which $k^{(\alpha)}-k^{(\beta)}+k^{(\gamma)} \notin\left\{k^{(1)}, \ldots, k^{(N)}\right\}+\mathbb{Z}^{d}$. Secondly, the multiplication of $U_{\text {app }}$ by $W$ generates terms with new concentration points, namely when $l^{(m)}+k^{(j)} \notin\left\{k^{(1)}, \ldots, k^{(N)}\right\}+\mathbb{Z}^{d}$ for some $m \in\left\{-m_{*}, \ldots, m_{*}\right\}, j \in\{1, \ldots, N\}$. These terms in $I$ and in $W u^{\text {app }}$ appear at the leading order and need to be eliminated in order to achieve a small residual. For this we define

$$
J:=J_{0} \cup J_{N} \cup J_{W}
$$

where

$$
\begin{aligned}
J_{0} & :=\left\{k^{(1)}, \ldots, k^{(N)}\right\}, \\
J_{N} & :=\left[\left(\left\{k^{(\alpha)}-k^{(\beta)}+k^{(\gamma)} \mid(\alpha, \beta, \gamma) \in\{1, \ldots, N\}^{3}\right\}+\mathbb{Z}^{d}\right) \cap \mathbb{B}\right] \backslash J_{0}, \\
J_{W} & :=\left[\left(\left\{k^{(j)}+l^{(m)} \mid, j \in\{1, \ldots, N\}, m \in\left\{-m_{*}, \ldots, m_{*}\right\}\right\}+\mathbb{Z}^{d}\right) \cap \mathbb{B}\right] \backslash J_{0} .
\end{aligned}
$$

We also define

$$
I_{0}:=\left\{\left(n_{1}, k^{(1)}\right), \ldots,\left(n_{N}, k^{(N)}\right)\right\} .
$$

Clearly, $J$ is finite. The set $J_{N} \cap J_{W}$ consists of the new concentration points discussed above. Below we modify the formal ansatz $u^{\text {app }}$ to an extended ansatz $u^{\text {ext }}$ by including correction terms concentrated near points in $J_{N} \cup J_{W}$. This is done in the $\vec{U}$-variables, i.e. after the application of $\mathcal{D}$.

The equation for $\vec{U}$ is

$$
\left(\mathrm{i} \partial_{t}-\Omega(k)\right) \vec{U}(k, t)+\varepsilon \sum_{m=-m_{*}}^{m_{*}} M^{(m)}(k) \vec{U}\left(k-l^{(m)}, t\right)+\vec{F}(\vec{U}, \vec{U}, \vec{U})(k, t)=0, k \in \mathbb{B}, t>0
$$

where for all $i, j \in \mathbb{N}$

$$
\begin{aligned}
& \Omega_{j j}(k)=\omega_{j}(k), \Omega_{i j}=0 \text { if } i \neq j, \\
& M_{i j}^{(m)}(k)=-a_{m}\left\langle p_{j}\left(\cdot, k-l^{(m)}\right), p_{i}(\cdot, k)\right\rangle_{\mathbb{T}}, \text { and } \\
& F_{j}(\vec{U}, \vec{U}, \vec{U})(k):=-\left\langle\sigma(\cdot)\left(\tilde{u} *_{\mathbb{B}} \tilde{\bar{u}} *_{\mathbb{B}} \tilde{u}\right)(\cdot, k, t), p_{j}(\cdot, k)\right\rangle_{\mathbb{T}},
\end{aligned}
$$

and where $\tilde{u}(x, k, t)=\sum_{n \in \mathbb{N}} U_{n}(k, t) p_{n}(x, k)$.

As advertised above, our extended ansatz must contain terms supported near $l \in J_{0}$ as well as near every $l \in J_{N} \cup J_{W}$. We choose these $k$-supports compact with the radius $\varepsilon^{1 / 2}$ for the terms concentrated near $k^{(1)}, \ldots, k^{(N)}$ and consequently with the radius $3 \varepsilon^{1 / 2}$ for $J_{N}$. For simplicity the terms concentrated near $l \in J_{W}$ are chosen also with the support of radius $3 \varepsilon^{1 / 2}$ because $J_{W} \cap J_{N}$ is not necessarily empty. Hence, we define the compactly supported envelopes 
$\tilde{A}_{j}, j \in\{1, \ldots, N\}$ and $\tilde{A}_{n, l},(n, l) \in(\mathbb{N} \times J) \backslash\left\{\left(n_{1}, k^{(1)}\right), \ldots,\left(n_{N}, k^{(N)}\right)\right\}$. The supports are

$$
\begin{aligned}
& \operatorname{supp}\left(\tilde{A}_{j}(\cdot, T)\right) \subset B_{\varepsilon^{-1 / 2}}, j=1, \ldots, N, \\
& \operatorname{supp}\left(\tilde{A}_{n, l}(\cdot, T)\right) \subset B_{3 \varepsilon^{-1 / 2}},(n, l) \in(\mathbb{N} \times J) \backslash\left\{\left(n_{j}, k^{(j)}\right): j=1, \ldots, N\right\},
\end{aligned}
$$

where $B_{r}:=B_{r}(0) \subset \mathbb{R}^{d}$ is the ball of radius $r>0$ centered at zero.

This leads us to the following extended ansatz. For $k \in \mathbb{R}^{d}, t \in \mathbb{R}$ we set

$$
\begin{aligned}
& U_{n_{j}}^{\mathrm{ext}}(k, t)=\varepsilon^{1 / 2-d} \sum_{\eta \in \mathbb{Z}^{d}}\left[\sum_{\substack{m\{1, \ldots, N\} \\
n_{m}=n_{j}}} \tilde{A}_{m}\left(\frac{k-k^{(m)}+\eta}{\varepsilon}, T\right)+\varepsilon \sum_{\substack{l \in J \\
\left(n_{j}, l\right) \notin I_{0}}} \tilde{A}_{n_{j}, l}\left(\frac{k-l+\eta}{\varepsilon}, T\right)\right] e^{-\mathrm{i} \omega_{0} t}, \\
& \text { for } j=1, \ldots, N \text {, } \\
& U_{n}^{\mathrm{ext}}(k, t)=\varepsilon^{3 / 2-d} \sum_{\eta \in \mathbb{Z}^{d}} \sum_{l \in J} \tilde{A}_{n, l}\left(\frac{k-l+\eta}{\varepsilon}, T\right) e^{-\mathrm{i} \omega_{0} t}, \text { for } n \in \mathbb{N} \backslash I_{0} .
\end{aligned}
$$

Note that the $\eta$-sums ensure the $\mathbb{Z}^{d}$-periodicity of $U_{n}^{\text {ext }}(\cdot, t)$ on $\mathbb{R}^{d}$. Due to the assumption on the supports of $\tilde{A}_{j}(\cdot, T)$ and $\tilde{A}_{n, l}(\cdot, T)$, the extended ansatz is supported in compact neighborhoods of the points in $J$ and their $\mathbb{Z}^{d}$-shifts.

For the estimate of the residual it is convenient to separate the leading order part of the ansatz. Hence, we set

$$
\vec{U}^{\text {ext }, 0}(k, t):=\varepsilon^{1 / 2-d} e^{-\mathrm{i} \omega_{0} t} \sum_{\eta \in \mathbb{Z}^{d}} \sum_{j=1}^{N} \tilde{A}_{j}\left(\frac{k-k^{(j)}+\eta}{\varepsilon}, T\right) e_{n_{j}} \text { and } \vec{U}^{\text {ext }, 1}:=\vec{U}^{\text {ext }}-\vec{U}^{\text {ext }, 0} .
$$

Here $e_{n}$ is the $n$-th Euclidean unit vector in $\mathbb{R}^{\mathbb{N}}$. Note that $\vec{U}^{\operatorname{ext}, 0}(\cdot, t)$ is supported in $\cup_{j=1}^{N} B_{\varepsilon^{1 / 2}}\left(k^{(j)}\right)+\mathbb{Z}^{d}$ and $\vec{U}^{\text {ext, } 1}(\cdot, t)$ is supported in $\cup_{l \in J} B_{3 \varepsilon^{1 / 2}}(l)+\mathbb{Z}^{d}$.

With the assumption on the supports of the envelopes the $\eta$-sums in $(6.19)$ can be reduced to finite sums if we restrict $k$ to the periodicity cell $\mathbb{B}$, i.e. $k \in \mathbb{B}$. Let us namely define for any $k \in \mathbb{B}$ the set

$$
Z_{k}:=\left\{\eta \in \mathbb{Z}^{d}: k-\eta \in \overline{\mathbb{B}}\right\} .
$$

Then for each $\tilde{A}_{j}$-term, $j=1, \ldots, N$, the sum is over $\eta \in Z_{k^{(j)}}$ and for each $\tilde{A}_{n, l}$-term, $l \in J, n \in \mathbb{N}$, the sum is over $\eta \in Z_{l}$.

6.2. Estimate of the residual. The residual for the extended ansatz is

$$
\overrightarrow{\operatorname{Res}}(k, t):=\left(\mathrm{i} \partial_{t}-\Omega(k)\right) \vec{U}^{\mathrm{ext}}(k, t)+\varepsilon \sum_{m=-m_{*}}^{m_{*}} M^{(m)}(k) \vec{U}^{\mathrm{ext}}\left(k-l^{(m)}, t\right)+\vec{F}\left(\vec{U}^{\text {ext }}, \vec{U}^{\text {ext }}, \vec{U}^{\text {ext }}\right)(k, t) .
$$

The most complicated term is the nonlinearity $\vec{F}$. However, we need to understand its detailed structure only for the leading order part $\vec{F}\left(\vec{U}^{\text {ext }, 0}, \vec{U}^{\text {ext }, 0}, \vec{U}^{\text {ext }, 0}\right)$ because this generates the nonlinearity in the CMEs. The rest of $\vec{F}$ will be simply estimated. We have

$$
F_{n}\left(\vec{U}^{\mathrm{ext}, 0}, \vec{U}^{\mathrm{ext}, 0}, \vec{U}^{\mathrm{ext}, 0}\right)(k, t)=-\left\langle\sigma(\cdot)\left(\tilde{u}^{\mathrm{ext}, 0} *_{\mathbb{B}} \tilde{\tilde{u}}^{\mathrm{ext}, 0} *_{\mathbb{B}} \tilde{u}^{\mathrm{ext}, 0}\right)(\cdot, k, t), p_{n}(\cdot, k)\right\rangle_{\mathbb{T}}, n \in \mathbb{N},
$$

where

$$
\tilde{u}^{\text {ext }, 0}(x, k, t):=\sum_{n \in \mathbb{N}} U_{n}^{\text {ext }, 0}(k, t) p_{n}(x, k)=\varepsilon^{1 / 2-d} \sum_{j=1}^{N} \sum_{\eta \in \mathbb{Z}^{d}} \tilde{A}_{j}\left(\frac{k-k^{(j)}+\eta}{\varepsilon}, T\right) p_{n_{j}}(x, k) e^{-\mathrm{i} \omega_{0} t} .
$$


The Bloch transformation of a function $u(x)$ satisfies $\widetilde{\widetilde{u}}(x, k)=\overline{\widetilde{u}}(x,-k)$. We impose this condition on the ansatz $\tilde{u}^{\text {ext, }, 0}$. This translates to the condition $\overline{\tilde{A}}(K, T)=\overline{\tilde{A}}(-K, T)$, which is satisfied, in particular, if $\tilde{A}$ is based on the Fourier transform of $A$, see $(6.22)$. We get

$$
\widetilde{\bar{u}}^{\mathrm{ext}, 0}(x, k, t)=\varepsilon^{1 / 2-d} \sum_{j=1}^{N} \sum_{\eta \in \mathbb{Z}^{d}} \tilde{\bar{A}}_{j}\left(\frac{k+k^{(j)}-\eta}{\varepsilon}, T\right) \overline{p_{n_{j}}}(x,-k) e^{\mathrm{i} \omega_{0} t} .
$$

A direct calculation leads to

$$
F_{n}\left(\vec{U}^{\mathrm{ext}, 0}, \vec{U}^{\mathrm{ext}, 0}, \vec{U}^{\mathrm{ext}, 0}\right)(k, t)=\varepsilon^{3 / 2-d} \sum_{l \in J_{N} \cup J_{0}} \sum_{(\alpha, \beta, \gamma) \in \Lambda_{l}} f_{n}^{\alpha, \beta, \gamma}(k, t)
$$

where

$$
\Lambda_{l}:=\left\{(\alpha, \beta, \gamma) \in\{1, \ldots, N\}^{3}: k^{(\alpha)}-k^{(\beta)}+k^{(\gamma)} \in l+\mathbb{Z}^{d}\right\}
$$

is the set of those indices $\alpha, \beta, \gamma$, for which the convolution term is concentrated at $l$,

$$
\begin{aligned}
f_{n}^{\alpha, \beta, \gamma}(k, t) & :=e^{-\mathrm{i} \omega_{0} t} \int_{B_{2 \varepsilon^{-1 / 2}}} \int_{B_{\varepsilon^{-1 / 2}}} \sum_{\eta \in \mathbb{Z}^{d}} \tilde{A}_{\alpha}\left(\frac{k-\left(k^{(\alpha)}-k^{(\beta)}+k^{(\gamma)}\right)+\eta-\varepsilon r}{\varepsilon}, T\right) \tilde{\bar{A}}_{\beta}(r-s, T) \times \\
& \times \tilde{A}_{\gamma}(s, T) b_{\alpha, \beta, \gamma}^{(n)}\left(k-\left(k^{(\gamma)}-k^{(\beta)}\right)-\varepsilon r, k^{(\beta)}-\varepsilon(r-s), k^{(\gamma)}+\varepsilon s, k\right) \mathrm{d} s \mathrm{~d} r \\
& \text { for } k \in B_{3 \varepsilon^{1 / 2}}\left(k^{(\alpha)}-k^{(\beta)}+k^{(\gamma)}\right), \\
b_{\alpha, \beta, \gamma}^{(n)}(q, r, s, k) & :=-\left\langle\sigma(\cdot) p_{n_{\alpha}}(\cdot, q) \overline{p_{n_{\beta}}}(\cdot, r) p_{n_{\gamma}}(\cdot, s), p_{n}(\cdot, k)\right\rangle_{\mathbb{T}}, \text { for } q, r, s, k \in \mathbb{R}^{d} .
\end{aligned}
$$

We consider the residual separately for $(n, k)$ with $n=n_{j}$ and $k$ near $k^{(j)}$ (or its integer shifts) with $j=1, \ldots, N$ and for other $(n, k) \in \mathbb{N} \times \mathbb{R}^{d}$.

Firstly, for $k \in B_{3 \varepsilon^{1 / 2}}\left(k^{(j)}\right), j=1, \ldots, N$ we have

$$
\begin{aligned}
& \operatorname{Res}_{n_{j}}(k, t)=\varepsilon^{3 / 2-d}\left[\mathrm{i} \partial_{T} \tilde{A}_{j}\left(\frac{k-k^{(j)}}{\varepsilon}, T\right)+\varepsilon^{-1}\left(\omega_{0}-\omega_{n_{j}}(k)\right) \tilde{A}_{j}\left(\frac{k-k^{(j)}}{\varepsilon}, T\right)\right. \\
& +\sum_{r=1}^{N} \sum_{\substack{m \in\left\{-m_{*}, \ldots, m_{*}\right\} \\
k^{(r)}+l^{(m)} \in k^{(j)}+\mathbb{Z}^{d}}} M_{n_{j}, n_{r}}^{(m)}(k) \tilde{A}_{r}\left(\frac{k-k^{(j)}}{\varepsilon}, T\right) \\
& -\sum_{(\alpha, \beta, \gamma) \in \Lambda_{k}(j)} \int_{B_{2 \varepsilon^{-1 / 2}}} \int_{B_{\varepsilon^{-1 / 2}}} \tilde{A}_{\alpha}\left(\frac{k-k^{(j)}-\varepsilon r}{\varepsilon}, T\right) \tilde{\bar{A}}_{\beta}(r-s, T) \tilde{A}_{\gamma}(s, T) \times \\
& \left.\times b_{\alpha, \beta, \gamma}^{\left(n_{j}\right)}\left(k-\left(k^{(\gamma)}-k^{(\beta)}\right)-\varepsilon r, k^{(\beta)}-\varepsilon(r-s), k^{(\gamma)}+\varepsilon s, k\right) \mathrm{d} s \mathrm{~d} r\right] e^{-\mathrm{i} \omega_{0} t}+\text { h.o.t. }
\end{aligned}
$$

We can recover $\operatorname{Res}_{n_{j}}(k, t)$ in the neighborhood of $k^{(j)}+\mathbb{Z}^{d}$ by the $\mathbb{Z}^{d}$ periodicity in $k$.

Secondly, for $(n, k) \in\left(\mathbb{N} \times \mathbb{R}^{d}\right) \backslash \cup_{j=1}^{N}\left(n_{j}, B_{3 \varepsilon^{1 / 2}}\left(k^{(j)}\right)+\mathbb{Z}^{d}\right)$

$$
\begin{aligned}
& \operatorname{Res}_{n}(k, t)=\varepsilon^{3 / 2-d}\left[\left(\omega_{0}-\omega_{n}(k)\right) \sum_{l \in J, \eta \in \mathbb{Z}^{d}} \tilde{A}_{n, l}\left(\frac{k-l+\eta}{\varepsilon}, T\right)\right. \\
& \quad+\sum_{j=1}^{N} \sum_{\substack{m=-m_{*}, \ldots, m_{*} \\
\eta \in \mathbb{Z}^{d}}} M_{n, n_{j}}^{(m)}(k) \tilde{A}_{j}\left(\frac{k-k^{(j)}-l^{(m)}+\eta}{\varepsilon}, T\right) \\
& \left.\quad+\sum_{l \in J_{N} \cup J_{0}} \sum_{(\alpha, \beta, \gamma) \in \Lambda_{l}} f_{n}^{\alpha, \beta, \gamma}(k, t)\right] e^{-\mathrm{i} \omega_{0} t}+\text { h.o.t.. }
\end{aligned}
$$


The higher order terms h.o.t. above come from the $T$-derivative of $\vec{U}^{\text {ext, } 1}$, from the application of the potential $W$ to $\vec{U}^{\text {ext,1 }}$ and from the nonlinearity $F_{n}$ with at least one of the three arguments being $\vec{U}^{\text {ext, } 1 \text {. }}$

1) $\operatorname{Res}_{n_{j}}(k, t)$ for $k \in B_{\varepsilon^{1 / 2}}\left(k^{(j)}\right), j=1, \ldots, N$

In order to ensure the smallness of $\operatorname{Res}_{n_{j}}$, we set

$$
\tilde{A}_{j}(K, T):=\chi_{B_{\varepsilon^{-1 / 2}}}(K) \widehat{A}_{j}(K, T), j \in\{1, \ldots, N\},
$$

where $\left(A_{1}, \ldots, A_{N}\right)$ solves 1.3 . This makes sense because, as we show next, the leading order part of $\operatorname{Res}_{n_{j}}$ is approximated by the Fourier transform of the left hand side of the $j$-th equation in (1.3). Let us start with the linear terms. For $k \in B_{\varepsilon^{1 / 2}}\left(k^{(j)}\right)$ we have

$$
\left|\omega_{n_{j}}(k)-\omega_{0}-\varepsilon \frac{k-k^{(j)}}{\varepsilon} \cdot v_{g}^{(j)}\right| \leqslant c \varepsilon^{2}\left|\frac{k-k^{(j)}}{\varepsilon}\right|^{2}\left(1+h_{\varepsilon}(k)\right)
$$

with $h_{\varepsilon}(k) \rightarrow 0$ for $\varepsilon \rightarrow 0$. Hence

$$
\begin{aligned}
& \int_{\mathbb{B}+k^{(j)}}\left|\varepsilon^{-1}\left(\omega_{0}-\omega_{n_{j}}\left(k^{(j)}\right)\right) \tilde{A}_{j}\left(\frac{k-k^{(j)}}{\varepsilon}, T\right)-\chi_{j}(k) \frac{k-k^{(j)}}{\varepsilon} \cdot v_{g}^{(j)} \widehat{A}_{j}\left(\frac{k-k^{(j)}}{\varepsilon}, T\right)\right| \mathrm{d} k \\
& \leqslant c \varepsilon^{1+d}\left\|\widehat{A}_{j}(\cdot, T)\right\|_{L_{2}^{1}\left(B_{\varepsilon^{-1 / 2}}(0)\right)} \leqslant c \varepsilon^{1+d}\left\|\widehat{A}_{j}(\cdot, T)\right\|_{L_{2}^{1}\left(\mathbb{R}^{d}\right)},
\end{aligned}
$$

where

$$
\chi_{j}:=\chi_{B_{\varepsilon^{1 / 2}\left(k^{(j)}\right)}}
$$

Next, we compare the linear coupling terms. For $j, r \in\{1, \ldots, N\}$ let $m \in\left\{-m_{*}, \ldots, m_{*}\right\}$ be such that $k^{(r)}+l^{(m)} \in k^{(j)}+\mathbb{Z}^{d}$. We rewrite in $M_{n_{j}, n_{r}}^{(m)}(k)$ the variables as

$$
k-l^{(m)}=k^{(r)}+\varepsilon \frac{k-k^{(j)}}{\varepsilon}+k^{(j)}-k^{(r)}-l^{(m)}
$$

and

We define

$$
k=k^{(j)}+\varepsilon \frac{k-k^{(j)}}{\varepsilon} .
$$

$$
K:=\frac{k-k^{(j)}}{\varepsilon} \text { for } k \in B_{\varepsilon^{1 / 2}}\left(k^{(j)}\right), j=1, \ldots, N,
$$

and write $\kappa_{j r}=\sum_{\substack{m \in\left\{-m_{*}, \ldots, m_{*}\right\} \\ k^{(r)}+l^{(m)} \in k^{(j)}+\mathbb{Z}^{d}}} \kappa_{j r}^{(m)}$, where

$$
\kappa_{j r}^{(m)}:=-a_{m} \int_{\mathbb{T}} e^{\mathrm{i}\left(k^{(r)}+l^{(m)}-k^{(j)}\right) \cdot x} p_{r}(x) \overline{p_{j}}(x) \mathrm{d} x .
$$

Then for $k \in B_{\varepsilon^{1 / 2}}\left(k^{(j)}\right)$ and $m \in\left\{-m_{*}, \ldots, m_{*}\right\}$ such that $k^{(r)}+l^{(m)} \in k^{(j)}+\mathbb{Z}^{d}$ it is

$$
\begin{aligned}
\left|M_{n_{j}, n_{r}}^{(m)}(k)-\kappa_{j r}^{(m)}\right| \leqslant\left|a_{m}\right| \int_{\mathbb{T}} \mid e^{\mathrm{i}\left(k^{(r)}-k^{(j)}+l^{(m)}\right) \cdot x} p_{n_{r}}\left(x, k^{(r)}+\varepsilon K\right) \overline{p_{n_{j}}}\left(x, k^{(j)}+\varepsilon K\right) & -e^{\mathrm{i}\left(k^{(r)}+l^{(m)}-k^{(j)}\right) \cdot x} p_{n_{r}}\left(x, k^{(r)}\right) \overline{p_{n_{j}}}\left(x, k^{(j)}\right) \mid \mathrm{d} x \\
& \leqslant\left|a_{m}\right| \int_{\mathbb{T}}\left|p_{n_{r}}\left(x, k^{(r)}+\varepsilon K\right)\right|\left|p_{n_{j}}\left(x, k^{(j)}+\varepsilon K\right)-p_{n_{j}}\left(x, k^{(j)}\right)\right| \mathrm{d} x \\
& +\left|a_{m}\right| \int_{\mathbb{T}}\left|p_{n_{j}}\left(x, k^{(j)}\right)\right|\left|p_{n_{r}}\left(x, k^{(r)}+\varepsilon K\right)-p_{n_{r}}\left(x, k^{(r)}\right)\right| \mathrm{d} x \\
& \leqslant c \varepsilon K
\end{aligned}
$$

due to (A6). 
From 6.24 we get

$$
\left\|M_{n_{j}, n_{r}}^{(m)}(\cdot) \tilde{A}_{r}\left(\frac{\cdot-k^{(j)}}{\varepsilon}, T\right)-\kappa_{j, r}^{(m)} \chi_{j} \widehat{A}_{r}\left(\frac{\cdot-k^{(j)}}{\varepsilon}, T\right)\right\|_{L^{1}\left(\mathbb{B}+k^{(j)}\right)} \leqslant c \varepsilon^{d+1}\left\|\widehat{A}_{r}(\cdot, T)\right\|_{L_{1}^{1}\left(\mathbb{R}^{d}\right)} .
$$

For the nonlinearity in the leading order part of $\operatorname{Res}_{n_{j}}(k, t)$ with $k \in B_{\varepsilon^{1 / 2}}\left(k^{(j)}\right)$ we need to estimate

$$
\begin{aligned}
\Psi_{j}^{\alpha, \beta, \gamma}(k, T):= & \int_{B_{2 \varepsilon^{-1 / 2}}} \int_{B_{\varepsilon^{-1 / 2}}} \tilde{A}_{\alpha}\left(\frac{k-k^{(j)}-\varepsilon r}{\varepsilon}, T\right) \tilde{\bar{A}}_{\beta}(r-s, T) \tilde{A}_{\gamma}(s, T) \times \\
& \times b_{\alpha, \beta, \gamma}^{\left(n_{j}\right)}\left(k-\left(k^{(\gamma)}-k^{(\beta)}\right)-\varepsilon r, k^{(\beta)}-\varepsilon(r-s), k^{(\gamma)}+\varepsilon s, k\right) \mathrm{d} s \mathrm{~d} r \\
& -\chi_{j} \gamma_{j}^{(\alpha, \beta, \gamma)}\left(\widehat{A}_{\alpha} * \widehat{\bar{A}}_{\beta} * \widehat{A}_{\gamma}\right)\left(\frac{k-k^{(j)}}{\varepsilon}, T\right)
\end{aligned}
$$

for each $(\alpha, \beta, \gamma) \in \Lambda_{k^{(j)}}$. We write

$$
\Psi_{j}^{\alpha, \beta, \gamma}=I_{1}+I_{2}+I_{3},
$$

where (using $\left.\gamma_{j}^{(\alpha, \beta, \gamma)}=b_{\alpha, \beta, \gamma}^{\left(n_{j}\right)}\left(k^{(\alpha)}, k^{(\beta)}, k^{(\gamma)}, k^{(j)}\right)\right)$

$$
\begin{aligned}
I_{1}:= & \int_{B_{2 \varepsilon^{-1 / 2}}} \int_{B_{\varepsilon^{-1 / 2}}} \tilde{A}_{\alpha}\left(\frac{k-k^{(j)}-\varepsilon r}{\varepsilon}, T\right) \tilde{\bar{A}}_{\beta}(r-s, T) \tilde{A}_{\gamma}(s, T) \times \\
\times & {\left[b_{\alpha, \beta, \gamma}^{\left(n_{j}\right)}\left(k-\left(k^{(\gamma)}-k^{(\beta)}\right)-\varepsilon r, k^{(\beta)}-\varepsilon(r-s), k^{(\gamma)}+\varepsilon s, k\right)\right.} \\
& \left.\quad-b_{\alpha, \beta, \gamma}^{\left(n_{j}\right)}\left(k^{(\alpha)}, k^{(\beta)}, k^{(\gamma)}, k^{(j)}\right)\right] \mathrm{d} s \mathrm{~d} r \\
I_{2}:= & \gamma_{j}^{(\alpha, \beta, \gamma)}\left[\left(\tilde{A}_{\alpha} *_{B_{2 \varepsilon^{-1 / 2}}} \tilde{\bar{A}}_{\beta} *_{\varepsilon^{-1 / 2}} \tilde{A}_{\gamma}\right)\left(\frac{k-k^{(j)}-\varepsilon r}{\varepsilon}, T\right)\right. \\
& \left.\quad-\chi_{B_{3 \varepsilon^{1 / 2}}\left(k^{(j)}\right)}(k)\left(\widehat{A}_{\alpha} * \widehat{\bar{A}}_{\beta} * \widehat{A}_{\gamma}\right)\left(\frac{k-k^{(j)}}{\varepsilon}, T\right)\right],
\end{aligned}
$$

and

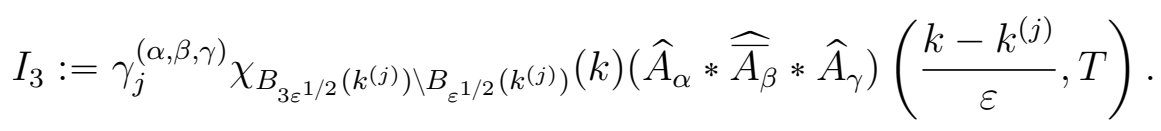

We estimate $\left\|I_{j}(\cdot, T)\right\|_{L^{1}\left(\mathbb{B}+k^{(j)}\right)}, j=1,2,3$ analogously to [7]. For $\left\|I_{1}(\cdot, T)\right\|_{L^{1}\left(\mathbb{B}+k^{(j)}\right)}$ we use the multilinearity of $b^{\left(n_{j}\right)}$ and the Lipschitz continuity of the Bloch functions $p_{n}(x, \cdot)$ in $(\mathrm{A} 6)$. $\left\|I_{2,3}(\cdot, T)\right\|_{L^{1}\left(\mathbb{B}+k^{(j)}\right)}$ are small due to the decay of $\widehat{A}_{j}(\cdot, T)$, namely due to

$$
\left\|\left(1-\chi_{B_{a \varepsilon}-1 / 2}\right) \widehat{A}_{j}(\cdot, T)\right\|_{L^{1}\left(\mathbb{R}^{d}\right)} \leqslant c_{a} \varepsilon^{\nu / 2}\left\|\widehat{A}_{j}(\cdot, T)\right\|_{L_{\nu}^{1}\left(\mathbb{R}^{d}\right)}
$$

for any $a, \nu>0$. One obtains

$$
\begin{aligned}
& \left\|I_{1}(\cdot, T)\right\|_{L^{1}\left(\mathbb{B}+k^{(j)}\right)} \leqslant c \varepsilon^{d+1}\left\|\widehat{A}_{\alpha}(\cdot, T)\right\|_{L_{1}^{1}\left(\mathbb{R}^{d}\right)}\left\|\widehat{A}_{\beta}(\cdot, T)\right\|_{L_{1}^{1}\left(\mathbb{R}^{d}\right)}\left\|\widehat{A}_{\gamma}(\cdot, T)\right\|_{L_{1}^{1}\left(\mathbb{R}^{d}\right)}, \\
& \left\|I_{2,3}(\cdot, T)\right\|_{L^{1}\left(\mathbb{B}+k^{(j)}\right)} \leqslant c \varepsilon^{d+\nu / 2}\left\|\widehat{A}_{\alpha}(\cdot, T)\right\|_{L_{\nu}^{1}\left(\mathbb{R}^{d}\right)}\left\|\widehat{A}_{\beta}(\cdot, T)\right\|_{L_{\nu}^{1}\left(\mathbb{R}^{d}\right)}\left\|\widehat{A}_{\gamma}(\cdot, T)\right\|_{L_{\nu}^{1}\left(\mathbb{R}^{d}\right)}
\end{aligned}
$$

for any $\nu>0$.

Estimates 6.23), 6.25) and (6.26) ensure that CMEs (1.3) yield a small $\operatorname{Res}_{n_{j}}(k, t), j=$ $1, \ldots, N$ for $k$ in a vicinity of $k^{(j)}+\mathbb{Z}^{d}$. The rest of the leading order part of Res is made small by a suitable choice of the correction terms $\tilde{A}_{n, l}, n \in \mathbb{N}, l \in J$.

2) Rest of Res at $O\left(\varepsilon^{3 / 2}\right)$ 
For $(n, k) \in \mathbb{N} \times \mathbb{R}^{d} \backslash \cup_{j=1}^{N}\left(n_{j}, B_{3 \varepsilon^{1 / 2}}\left(k^{(j)}\right)+\mathbb{Z}^{d}\right)$ the leading order $\left(O\left(\varepsilon^{3 / 2}\right)\right)$ part of the residual vanishes if we set

$$
\tilde{A}_{n, l}\left(\frac{k-l}{\varepsilon}, T\right):=\frac{1}{\omega_{0}-\omega_{n}(k)}\left[\sum_{\substack{j=1 \\ j}}^{N} \sum_{\substack{m \in\left\{-m_{*}, \ldots, m_{*}\right\} \\ k^{(j)}+l^{(m)} \in l+\mathbb{Z}^{d}}} M_{n, n_{j}}^{(m)} \tilde{A}_{j}\left(\frac{k-l}{\varepsilon}, T\right)-\sum_{(\alpha, \beta, \gamma) \in \Lambda_{l}} f_{n}^{\alpha, \beta, \gamma}(k, t)\right]
$$

for all $l \in J$ if $n \in \mathbb{N} \backslash\left\{n_{1}, \ldots, n_{N}\right\}$, and all $l \in J$ such that $\left(n_{j}, l\right) \notin I_{0}$ if $n=n_{j}, j=1, \ldots, N$.

Note that in 6.27) is

$$
\left|\omega_{0}-\omega_{n}(k)\right|>c n^{2 / d}, \quad n \in \mathbb{N}
$$

with some $c>0$. For $n \in\left\{n_{1}, \ldots, n_{N}\right\}$ this holds because $(n, l) \neq\left(n_{j}, k^{(j)}\right)$ and $k \in B_{3 \varepsilon^{1 / 2}}(l)$, and the growth in $n$ is guaranteed by (1.8).

Combining now 6.23, 6.25), and (6.26), we have

$$
\begin{aligned}
\|\operatorname{Res}(\cdot, t)\|_{\mathcal{X}(s)} \leqslant & \| \text { h.o.t. }(\cdot, t) \|_{\mathcal{X}(s)}+c \varepsilon^{\frac{5}{2}}\left(\sum_{\alpha \in\{1, \ldots, N\}}\left\|\widehat{A}_{\alpha}(\cdot, T)\right\|_{L_{2}^{1}\left(\mathbb{R}^{d}\right)}\right. \\
& \left.+\sum_{\alpha, \beta, \gamma \in\{1, \ldots, N\}}\left\|\widehat{A}_{\alpha}(\cdot, T)\right\|_{L_{2}^{1}\left(\mathbb{R}^{d}\right)}\left\|\widehat{A}_{\beta}(\cdot, T)\right\|_{L_{2}^{1}\left(\mathbb{R}^{d}\right)}\left\|\widehat{A}_{\gamma}(\cdot, T)\right\|_{L_{2}^{1}\left(\mathbb{R}^{d}\right)}\right) .
\end{aligned}
$$

Thus it remains to estimate the h.o.t..

The $n$-th component (h.o.t. $)_{n}(k, t)$ consists of a (finite) sum over $l$ of the terms $\varepsilon^{5 / 2-d} \partial_{T} \tilde{A}_{n, l}\left(\frac{k-l}{\varepsilon}, T\right) e^{-}$ and of $\varepsilon \sum_{m \in\left\{-m_{*}, \ldots, m_{*}\right\}}\left(M^{(m)}(k) \vec{U}^{\text {ext }, 1}\left(k-l^{(m)}, t\right)\right)_{n}, F_{n}\left(\vec{U}^{\text {ext }, 1}, \vec{U}^{\text {ext }, 0}, \vec{U}^{\text {ext }, 0}\right), F_{n}\left(\vec{U}^{\text {ext }, 0}, \vec{U}^{\text {ext }, 1}, \vec{U}^{\text {ext }, 0}\right)$ as well as of nonlinear terms quadratic or cubic in $\vec{U}^{\text {ext, } 1}$.

For $\varepsilon^{5 / 2-d} \partial_{T} \tilde{A}_{n, l}\left(\frac{k-l}{\varepsilon}, T\right) e^{-\mathrm{i} \omega_{0} t}$ we first note that

$$
\left|M_{n, n_{j}}^{(m)}\right|=\frac{\left|a_{m}\right|}{\left|\omega_{n}(k)\right|^{q}}\left\langle\mathcal{L}^{q}(\cdot, k) p_{n_{j}}\left(\cdot, k-l^{(m)}\right), p_{n}(\cdot, k)\right\rangle_{\mathbb{T}} \leqslant c n^{-2 q / d} \sup _{k \in \mathbb{B}}\left\|p_{n_{j}}(\cdot, k)\right\|_{H^{2 q}(\mathbb{T})}
$$

for any $q \in \mathbb{N}$. Similarly,

$$
\begin{aligned}
\left|b_{\alpha, \beta, \gamma}^{(n)}(o, r, s, k)\right| & =\frac{1}{\left|\omega_{n}(k)\right|^{q}}\left\langle\mathcal{L}^{q}(\cdot, k)\left(\sigma(\cdot) p_{n_{\alpha}}(\cdot, o) \overline{p_{n_{\beta}}}(\cdot, r) p_{n_{\gamma}}(\cdot, s)\right), p_{n}(\cdot, k)\right\rangle_{\mathbb{T}} \\
& \leqslant c n^{-2 q / d} \sup _{k \in \mathbb{B}}\left\|p_{n_{\alpha}}(\cdot, k)\right\|_{H^{2 q(\mathbb{T})}} \sup _{k \in \mathbb{B}}\left\|p_{n_{\beta}}(\cdot, k)\right\|_{H^{2 q(\mathbb{T})}} \sup _{k \in \mathbb{B}}\left\|p_{n_{\gamma}}(\cdot, k)\right\|_{H^{2 q(\mathbb{T})}}
\end{aligned}
$$

for all $o, r, s, k \in 2 \mathbb{B}$ and $q \in \mathbb{N} \cap\left(\frac{d}{4}, \infty\right)$ if $\sigma \in H^{2 q}(\mathbb{T})$.

The required $H^{2 q}$-regularity of the Bloch waves $p_{n_{j}}(\cdot, k), j=1, \ldots, N$ is satisfied if $V \in$ $H^{\mu}(\mathbb{T}), \mu>2 q+d-2$, see Lemma 3 in [7]. We obtain

$$
\begin{aligned}
\left\|\varepsilon^{5 / 2-d} \partial_{T} \tilde{A}_{n, l}\left(\frac{\cdot-l}{\varepsilon}, T\right)\right\|_{L^{1}(\mathbb{B})} \leqslant & c \varepsilon^{5 / 2} n^{-2(q+1) / d}\left(\sum_{j=1}^{N}\left\|\partial_{T} \widehat{A}_{j}(\cdot, T)\right\|_{L^{1}\left(\mathbb{R}^{d}\right)}\right. \\
& \left.+\sum_{\alpha, \beta, \gamma \in\{1, \ldots, N\}}\left\|\partial_{T} \widehat{A}_{\alpha}(\cdot, T)\right\|_{L^{1}\left(\mathbb{R}^{d}\right)}\left\|\widehat{A}_{\beta}(\cdot, T)\right\|_{L^{1}\left(\mathbb{R}^{d}\right)}\left\|\widehat{A}_{\gamma}(\cdot, T)\right\|_{L^{1}\left(\mathbb{R}^{d}\right)}\right)
\end{aligned}
$$


and

(6.29)

$$
\begin{aligned}
\| \varepsilon^{5 / 2-d}\left(\partial_{T} \tilde{A}_{n, l}\left(\frac{\cdot-l}{\varepsilon}, T\right)\right)_{n \in \mathbb{N}} & \|_{\mathcal{X}(s)} \leqslant c \varepsilon^{5 / 2}\left(\sum_{j=1}^{N}\left\|\partial_{T} \widehat{A}_{j}(\cdot, T)\right\|_{L^{1}\left(\mathbb{R}^{d}\right)}\right. \\
& \left.+\sum_{\alpha, \beta, \gamma \in\{1, \ldots, N\}}\left\|\partial_{T} \widehat{A}_{\alpha}(\cdot, T)\right\|_{L^{1}\left(\mathbb{R}^{d}\right)}\left\|\widehat{A}_{\beta}(\cdot, T)\right\|_{L^{1}\left(\mathbb{R}^{d}\right)}\left\|\widehat{A}_{\gamma}(\cdot, T)\right\|_{L^{1}\left(\mathbb{R}^{d}\right)}\right)
\end{aligned}
$$

provided $q>\frac{s}{2}+\frac{d}{4}-1$.

Next, we have

$$
\begin{aligned}
& \left\|\sum_{m \in\left\{-m_{*}, \ldots, m_{*}\right\}} M^{(m)}(\cdot) \vec{U}^{\text {ext }, 1}\left(\cdot-l^{(m)}, t\right)\right\|_{\mathcal{X}(s)} \leqslant c \varepsilon\left\|W \tilde{u}^{\text {ext }, 1}(\cdot, \cdot, t)\right\|_{L^{1}\left(\mathbb{B}, H^{s}(\mathbb{T})\right)} \\
& \leqslant c \varepsilon\left\|\tilde{u}^{\operatorname{ext}, 1}(\cdot, \cdot, t)\right\|_{L^{1}\left(\mathbb{B}, H^{s}(\mathbb{T})\right)} \leqslant c \varepsilon\left\|\vec{U}^{\operatorname{ext}, 1}(\cdot, t)\right\|_{\mathcal{X}(s)}
\end{aligned}
$$

For the nonlinear terms in h.o.t. we use the algebra property

$$
\left\|\tilde{u} *_{\mathbb{B}} \tilde{v}\right\|_{L^{1}\left(\mathbb{B}, H^{s}(\mathbb{T})\right)} \leqslant c\|\tilde{u}\|_{L^{1}\left(\mathbb{B}, H^{s}(\mathbb{T})\right)}\|\tilde{v}\|_{L^{1}\left(\mathbb{B}, H^{s}(\mathbb{T})\right)}
$$

if $s>d / 2$, see Lemma 2 in [7]. This yields, for instance

$$
\left\|\vec{F}\left(\vec{U}^{\text {ext }, 1}, \vec{U}^{\text {ext }, 0}, \vec{U}^{\text {ext }, 0}\right)\right\|_{\mathcal{X}(s)} \leqslant c\left\|\vec{U}^{\text {ext }, 1}(\cdot, t)\right\|_{\mathcal{X}(s)}\left\|\vec{U}^{\text {ext }, 0}(\cdot, t)\right\|_{\mathcal{X}(s)}^{2} .
$$

To make inequalities 6.30 and 6.32 useful, it remains to estimate $\left\|\vec{U}^{\text {ext, }, 0}(\cdot, t)\right\|_{\mathcal{X}(s)}$ and $\left\|\vec{U}^{\text {ext, } 1}(\cdot, t)\right\|_{\mathcal{X}(s)}$. We have

$$
\left\|\vec{U}^{\text {ext }, 0}(\cdot, t)\right\|_{\mathcal{X}(s)} \leqslant c \varepsilon^{1 / 2} \sum_{j=1}^{N}\left\|\widehat{A}_{j}(\cdot, T)\right\|_{L^{1}\left(\mathbb{R}^{d}\right)}
$$

and

$$
\begin{aligned}
\left\|\vec{U}^{\mathrm{ext}, 1}(\cdot, t)\right\|_{\mathcal{X}(s)} & =\left(\sum_{n \in \mathbb{N}} n^{2 s / d}\left\|U_{n}^{\mathrm{ext}, 1}(\cdot, t)\right\|_{L^{1}(\mathbb{B})}^{2}\right)^{1 / 2} \\
\leqslant & c \varepsilon^{3 / 2}\left(\sum_{n \in \mathbb{N}} n^{2 s / d-4(q+1) / d}\right)^{1 / 2}\left(\sum_{j=1}^{N}\left\|\widehat{A}_{j}(\cdot, T)\right\|_{L^{1}\left(\mathbb{R}^{d}\right)}\right. \\
& \left.+\sum_{\alpha, \beta, \gamma \in\{1, \ldots, N\}}\left\|\widehat{A}_{\alpha}(\cdot, T)\right\|_{L^{1}\left(\mathbb{R}^{d}\right)}\left\|\widehat{A}_{\beta}(\cdot, T)\right\|_{L^{1}\left(\mathbb{R}^{d}\right)}\left\|\widehat{A}_{\gamma}(\cdot, T)\right\|_{L^{1}\left(\mathbb{R}^{d}\right)}\right) \\
& \leqslant c \varepsilon^{3 / 2}\left(\sum_{j=1}^{N}\left\|\widehat{A}_{j}(\cdot, T)\right\|_{L^{1}\left(\mathbb{R}^{d}\right)}\right. \\
& \left.+\sum_{\alpha, \beta, \gamma \in\{1, \ldots, N\}}\left\|\widehat{A}_{\alpha}(\cdot, T)\right\|_{L^{1}\left(\mathbb{R}^{d}\right)}\left\|\widehat{A}_{\beta}(\cdot, T)\right\|_{L^{1}\left(\mathbb{R}^{d}\right)}\left\|\widehat{A}_{\gamma}(\cdot, T)\right\|_{L^{1}\left(\mathbb{R}^{d}\right)}\right)
\end{aligned}
$$

if, again, $q>\frac{s}{2}+\frac{d}{4}-1$. In 6.34 we used $\sigma \in H^{2 q}(\mathbb{T})$ and $V \in H^{\mu}(\mathbb{T}), \mu>2 q+d-2$, similarly to above, in order to get the decay in $n$. 
In summary, collecting 6.28), 6.29), 6.30), 6.32), 6.33) and (6.34) if $\sigma \in H^{2 q}(\mathbb{T}), V \in$ $H^{\mu}(\mathbb{T}), \mu>2 q+d-2, q \in \mathbb{N} \cap\left(\frac{s}{2}+\frac{d}{4}-1, \infty\right)$, and $\widehat{A}_{j}(\cdot, T) \in L_{2}^{1}\left(\mathbb{R}^{2}\right), \partial_{T} \widehat{A}_{j}(\cdot, T) \in L^{1}\left(\mathbb{R}^{2}\right)$ for all $T \in\left[0, T_{0}\right]$, then

$$
\|\overrightarrow{\operatorname{Res}}(\cdot, t)\|_{\mathcal{X}(s)} \leqslant c \varepsilon^{5 / 2} \quad \text { for all } t \in\left[0, T_{0} \varepsilon^{-1}\right],
$$

where $c>0$ depends polynomially on $\left\|\widehat{A}_{j}(\cdot, T)\right\|_{L_{2}^{1}\left(\mathbb{R}^{d}\right)}$ and $\left\|\partial_{T} \widehat{A}_{j}(\cdot, T)\right\|_{L^{1}\left(\mathbb{R}^{d}\right)}, j=1, \ldots, N$.

6.3. Estimation of the Error. Using the triangle inequality, we estimate the error $\|(u-$ $\left.u^{\text {app }}\right)(\cdot, t) \|_{C_{b}^{0}\left(\mathbb{R}^{d}\right)}$ by the sum of $\left\|\left(\vec{U}^{\text {ext }}-\vec{U}^{\text {app }}\right)(\cdot, t)\right\|_{\mathcal{X}(s)}$ and $\|\vec{E}(\cdot, t)\|_{\mathcal{X}(s)}$, where

$$
\vec{E}:=\vec{U}-\vec{U}^{\text {ext }} \text {. }
$$

We estimate $\left\|\left(\vec{U}^{\text {ext }}-\vec{U}^{\text {app }}\right)(\cdot, t)\right\|_{\mathcal{X}(s)}$ directly and $\|\vec{E}(\cdot, t)\|_{\mathcal{X}(s)}$ via Gronwall's inequality

Lemma 3. If $V \in H^{\mu}(\mathbb{T})$ and $\sigma \in H^{2 q}(\mathbb{T})$ with some $\mu>2 q+d-2, q \in \mathbb{N} \cap\left(\frac{s}{2}+\frac{d}{4}, \infty\right)$, and $\widehat{A}_{j}(\cdot, T) \in L_{\beta}^{1}\left(\mathbb{R}^{d}\right), j=1, \ldots, N$, with some $\beta>2 q+d$, then there is $c>0$ such that

$$
\begin{aligned}
\left\|\left(\vec{U}^{a p p}-\vec{U}^{e x t}\right)(\cdot, t)\right\|_{\mathcal{X}(s)} \leqslant & c\left(\varepsilon^{3 / 2} \sum_{j=1}^{N}\left\|\widehat{A}_{j}(\cdot, T)\right\|_{L_{\beta}^{1}\left(\mathbb{R}^{d}\right)}\right. \\
& \left.+\varepsilon^{1 / 2+2 d} \sum_{\alpha, \beta, \gamma \in\{1, \ldots, N\}}\left\|\widehat{A}_{\alpha}(\cdot, T)\right\|_{L^{1}\left(\mathbb{R}^{d}\right)}\left\|\widehat{A}_{\beta}(\cdot, T)\right\|_{L^{1}\left(\mathbb{R}^{d}\right)}\left\|\widehat{A}_{\gamma}(\cdot, T)\right\|_{L^{1}\left(\mathbb{R}^{d}\right)}\right)
\end{aligned}
$$

for all $\varepsilon>0$ small enough.

Proof. Because $\widehat{A}_{j}(\cdot, T) \in L^{2}\left(\mathbb{R}^{d}\right)$, we have also $A_{j}(\cdot, T) \in L^{2}\left(\mathbb{R}^{d}\right)$ leading to $u^{\text {app }}(\cdot, t) \in L^{2}\left(\mathbb{R}^{d}\right)$. Therefore, it makes sense to evaluate $\mathcal{D} \mathcal{T} u^{\text {app }}(\cdot, t)$, producing

$$
U_{n}^{\mathrm{app}}(k, t)=\varepsilon^{1 / 2-d} \sum_{j=1}^{N} \sum_{\eta \in \mathbb{Z}^{d}} \widehat{A}_{j}\left(\frac{k-k^{(j)}+\eta}{\varepsilon}, T\right)\left\langle p_{n_{j}}\left(\cdot, k^{(j)}-\eta\right), p_{n}(\cdot, k)\right\rangle_{L^{2}(\mathbb{T})}, n \in \mathbb{N} .
$$

It is

$$
\begin{aligned}
\left\|\left(\vec{U}^{\mathrm{app}}-\vec{U}^{\mathrm{ext}}\right)(\cdot, t)\right\|_{\mathcal{X}(s)} \leqslant & c \sum_{j=1}^{N}\left\|\left(U_{n_{j}}^{\mathrm{app}}-U_{n_{j}}^{\mathrm{ext}, 0}\right)(\cdot, t)\right\|_{L^{1}(\mathbb{B})}+\left\|\left(U_{n}^{\mathrm{app}}(\cdot, t)\right)_{n \in \mathbb{N} \backslash\left\{n_{1}, \ldots, n_{N}\right\}}\right\|_{\mathcal{X}(s)} \\
& +\left\|\vec{U}^{\mathrm{ext}, 1}(\cdot, t)\right\|_{\mathcal{X}(s)} .
\end{aligned}
$$

$\left\|\vec{U}^{\text {ext }, 1}(\cdot, t)\right\|_{\mathcal{X}(s)}$ is estimated in $(6.34)$. The remaining two contributions are estimated in a complete analogy to Lemma 10 in [7]. We get

$$
\left\|\left(U_{n_{j}}^{\text {app }}-U_{n_{j}}^{\text {ext,0}}\right)(\cdot, t)\right\|_{L^{1}(\mathbb{B})} \leqslant c\left(\varepsilon^{3 / 2}\left\|\widehat{A}_{j}(\cdot, T)\right\|_{L_{1}^{1}\left(\mathbb{R}^{d}\right)}+\varepsilon^{1 / 2+\nu / 2}\left\|\widehat{A}_{j}(\cdot, T)\right\|_{L_{\nu}^{1}\left(\mathbb{R}^{d}\right)}\right)
$$

for any $\nu>0$ using the Lipschitz continuity $\left|\left\langle p_{n_{j}}\left(\cdot, k^{(j)}\right), p_{n_{j}}\left(\cdot, k^{(j)}\right)-p_{n_{j}}(\cdot, k)\right\rangle_{L^{2}(\mathbb{T})}\right| \leqslant L\left|k-k^{(j)}\right|$ guaranteed by (A6).

Finally,

$$
\left\|\left(U_{n}^{\mathrm{app}}(\cdot, t)\right)_{n \in \mathbb{N} \backslash\left\{n_{1}, \ldots, n_{N}\right\}}\right\|_{\mathcal{X}(s)} \leqslant c\left(\varepsilon^{3 / 2} \sum_{j=1}^{N}\left\|\widehat{A}_{j}(\cdot, T)\right\|_{L_{1}^{1}\left(\mathbb{R}^{d}\right)}+\varepsilon^{1 / 2+\beta} \sum_{j=1}^{N}\left\|\widehat{A}_{j}(\cdot, T)\right\|_{L_{\beta}^{1}\left(\mathbb{R}^{d}\right)}\right)
$$

provided $\beta>2 q+d, q \in \mathbb{N} \cap\left(\frac{s}{2}+\frac{d}{4}, \infty\right), V \in H^{a}(\mathbb{T}), a>2 q+d-2$. This estimate uses the Lipschitz continuity of $p_{n_{j}}(x, \cdot)$, the growth of the eigenvalues $\omega_{n}$, and the fact $\| \mathcal{L}^{q}(k) p_{n_{0}}(\cdot, k-$ $\eta) \|_{L^{2}(\mathbb{T})} \leqslant c|\eta|^{2 q}$. For details see Lemma 10 in [7]. 
The factor $\varepsilon^{1 / 2+2 d}$ in the Lemma comes from $(6.36)$ and $\beta>2 q+d, q>s / 2+d / 4, s>d / 2$, which implies $\beta>2 d$.

Let us consolidate the regularity assumptions needed in the proof of Theorem 1 . The estimates are performed in $\mathcal{X}(s), s>d / 2$. The residual estimate 6.35 and Lemma 3 require $\hat{A}_{j} \in$ $C\left(\left[0, T_{0}\right], L_{\beta}^{1}\left(\mathbb{R}^{d}\right) \cap L^{2}\left(\mathbb{R}^{d}\right)\right), \partial_{T} \widehat{A}_{j} \in C\left(\left[0, T_{0}\right], L^{1}\left(\mathbb{R}^{d}\right)\right)$ for some $\beta>2 q+d, q \in \mathbb{N} \cap\left(\frac{s}{2}+\frac{d}{4}, \infty\right)$. We can choose $q=\left\lceil\frac{d}{2}\right\rceil+1$ with $s \in\left(\frac{d}{2}, 2\left(\left\lceil\frac{d}{2}\right\rceil+1\right)-\frac{d}{2}\right)$. Then the potentials need to satisfy $\left.\sigma \in H^{2}\right\rceil^{\left.\frac{d}{2}\right\rceil+2}(\mathbb{T})$ and $V \in H^{\mu}(\mathbb{T})$ with $\mu>2\left\lceil\frac{d}{2}\right\rceil+d$. In summary, the regularity conditions are

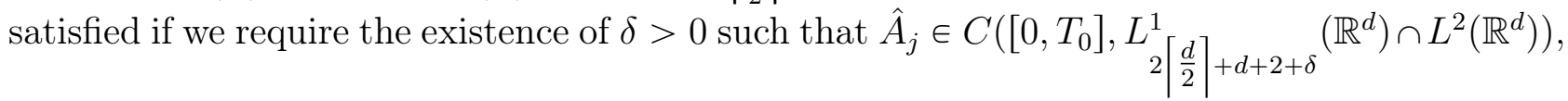

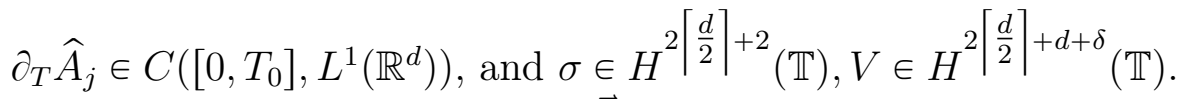

Finally, we estimate the error $\vec{E}$. The procedure is completely analogous to that in Sec. 4 of [6]. We present it here for completeness. The error satisfies

$$
\partial_{t} \vec{E}=-\mathrm{i} \Omega(k) \vec{E}+\mathrm{i} \vec{G}\left(\vec{U}^{\text {ext }}, \vec{E}\right)
$$

with

$$
\vec{G}\left(\vec{U}^{\text {ext }}, \vec{E}\right)=\overrightarrow{\operatorname{Res}}+\vec{F}(\vec{U}, \vec{U}, \vec{U})-\vec{F}\left(\vec{U}^{\text {ext }}, \vec{U}^{\text {ext }}, \vec{U}^{\text {ext }}\right)-\varepsilon \sum_{m=-m_{*}}^{m_{*}} M^{(m)} \vec{E}\left(\cdot+l^{(m)}, t\right) .
$$

Due to the cubic structure of $\vec{F}$, and the isomorphism property of $\mathcal{D}$ and the algebra property 6.31) we have for $s>d / 2$ the existence of $c>0$ such that

$$
\left\|\vec{F}(\vec{U}, \vec{U}, \vec{U})-\vec{F}\left(\vec{U}^{\text {ext }}, \vec{U}^{\text {ext }}, \vec{U}^{\text {ext }}\right)\right\|_{\mathcal{X}(s)} \leqslant c\left(\left\|\vec{U}^{\text {ext }}\right\|_{\mathcal{X}(s)}^{2}\|\vec{E}\|_{\mathcal{X}(s)}+\left\|\vec{U}^{\text {ext }}\right\|\left\|_{\mathcal{X}(s)}\right\| \vec{E}\left\|_{\mathcal{X}(s)}^{2}+\right\| \vec{E} \|_{\mathcal{X}(s)}^{3}\right) .
$$

We also have

$$
\left\|\varepsilon \sum_{m=-m_{*}}^{m_{*}} M^{(m)}(\cdot) \vec{E}\left(\cdot+l^{(m)}, t\right)\right\|_{\mathcal{X}(s)} \leqslant c \varepsilon\|\vec{E}(\cdot, t)\|_{\mathcal{X}(s)} .
$$

Next, using (6.33) and (6.34), we get

$$
\left\|\vec{U}^{\text {ext }}(\cdot, t)\right\|_{\mathcal{X}(s)} \leqslant c \varepsilon^{1 / 2} \quad \text { for all } t \in\left[0, \varepsilon^{-1} T_{0}\right] .
$$

Hence, there are $c_{1}, c_{2}, c_{3}>0$ such that

$$
\left\|\vec{G}\left(\vec{U}^{\text {ext }}, \vec{E}\right)(t)\right\|_{\mathcal{X}(s)} \leqslant c_{1} \varepsilon\|\vec{E}(t)\|_{\mathcal{X}(s)}+c_{2} \varepsilon^{1 / 2}\|\vec{E}(t)\|_{\mathcal{X}(s)}^{2}+c_{3}\|\vec{E}(t)\|_{\mathcal{X}(s)}^{3}+C_{\operatorname{Res}} \varepsilon^{5 / 2}
$$

for all $t \in\left[0, \varepsilon^{-1} T_{0}\right]$.

We rewrite equation (6.37) as

$$
\vec{E}(t)=\vec{E}(0)+\int_{0}^{t} S(t-\tau) \vec{G}\left(\vec{U}^{\mathrm{ext}}, \vec{E}\right)(\tau) d \tau
$$

where $S(t)=e^{-\mathrm{i} \Omega t}: \mathcal{X}(s) \rightarrow \mathcal{X}(s)$ is a strongly continuous unitary group generated by $-\mathrm{i} \Omega(k)$. Hence, we have

$$
\|\vec{E}(t)\|_{\mathcal{X}(s)} \leqslant\|\vec{E}(0)\|_{\mathcal{X}(s)}+\int_{0}^{t} c_{1} \varepsilon\|\vec{E}(\tau)\|_{\mathcal{X}(s)}+c_{2} \varepsilon^{1 / 2}\|\vec{E}(\tau)\|_{\mathcal{X}(s)}^{2}+c_{3}\|\vec{E}(\tau)\|_{\mathcal{X}(s)}^{3}+C_{\operatorname{Res}} \varepsilon^{5 / 2} d \tau .
$$


Because $\vec{U}(0)=\vec{U}^{\text {app }}(0)$, we get $\vec{E}(0)=\vec{U}^{\text {app }}(0)-\vec{U}^{\text {ext }}(0)$ and Lemma 3 provides $\|\vec{E}(0)\|_{\mathcal{X}(s)} \leqslant$ $C_{0} \varepsilon^{3 / 2}$ for all $\varepsilon \in\left(0, \varepsilon_{0}\right)$ with some $\varepsilon_{0}>0$.

Given an $M>C_{0}$ there exists $T_{*}>0$ such that $\|\vec{E}(t)\|_{\mathcal{X}(s)} \leqslant M \varepsilon^{3 / 2}$ for all $t \in\left[0, T_{*}\right]$. In the following, using Gronwall's inequality and a bootstrapping argument, we choose $\varepsilon_{0}>0$ and $M>0$ such that if $\varepsilon \in\left(0, \varepsilon_{0}\right)$, then $\|\vec{E}(t)\|_{\mathcal{X}(s)} \leqslant M \varepsilon^{3 / 2}$ for all $t \in\left[0, \varepsilon^{-1} T_{0}\right]$.

If $\|\vec{E}(t)\|_{\mathcal{X}(s)} \leqslant M \varepsilon^{3 / 2}$, then

$$
\begin{aligned}
\|\vec{E}(t)\|_{\mathcal{X}(s)} & \leqslant C_{0} \varepsilon^{3 / 2}+\int_{0}^{t} c_{1} \varepsilon\|\vec{E}(\tau)\|_{\mathcal{X}(s)} d \tau+t\left(c_{2} \varepsilon^{7 / 2} M^{2}+c_{3} \varepsilon^{9 / 2} M^{3}+C_{\mathrm{Res}} \varepsilon^{5 / 2}\right) \\
& \leqslant \varepsilon^{3 / 2}\left[C_{0}+t \varepsilon\left(c_{2} \varepsilon M^{2}+c_{3} \varepsilon^{2} M^{3}+C_{\mathrm{Res}}\right)\right] e^{c_{1} \varepsilon t}
\end{aligned}
$$

where the second step follows by Gronwall's inequality. For the desired estimate on $t \in$ $\left[0, \varepsilon^{-1} T_{0}\right]$, we redefine

$$
M:=C_{0}+T_{0}\left(C_{\text {Res }}+1\right) e^{c_{1} T_{0}}
$$

and choose $\varepsilon_{0}$ so small that $c_{2} \varepsilon_{0} M^{2}+c_{3} \varepsilon_{0}^{2} M^{3} \leqslant 1$. Then, clearly,

$$
\sup _{t \in\left[0, \varepsilon^{-1} T_{0}\right]}\|\vec{E}(t)\|_{\mathcal{X}(s)} \leqslant M \varepsilon^{3 / 2} .
$$

This completes the proof of Theorem (1).

\section{ACKNOWLEDGMENTS}

This research is supported by the German Research Foundation, DFG grant No. DO1467/31 .

\section{REFERENCES}

[1] M.J. Ablowitz and Z.H. Musslimani. Spectral renormalization method for computing self-localized solutions to nonlinear systems. Opt. Lett., 30(16):2140-2142, 2005.

[2] A. B. Aceves and S. Wabnitz. Self induced transparency solitons in nonlinear refractive media. Phys. Lett. A, 141:37-42, 1989 .

[3] $\overline{\mathrm{D}}$. Agueev and D. Pelinovsky. Modeling of wave resonances in low-contrast photonic crystals. SIAM Journal on Applied Mathematics, 65(4):1101-1129, 2005.

[4] K. Busch, G. Schneider, L. Tkeshelashvili, and H. Uecker. Justification of the nonlinear Schrödinger equation in spatially periodic media. Z. Angew. Math. Phys., 57:905-939, 2006.

[5] T. Dohnal. Traveling Solitary Waves in the Periodic Nonlinear Schrödinger Equation with Finite Band Potentials. SIAM J. Appl. Math., 74:306-321, 2014.

[6] T. Dohnal and L. Helfmeier. Justification of the Coupled Mode Asymptotics for Localized Wavepackets in the Periodic Nonlinear Schrödinger Equation. J. Math. Anal. Appl., 450:691-726, 2017.

[7] T. Dohnal and D. Rudolf. NLS Approximation for Wavepackets in Periodic Cubically Nonlinear Wave Problems in $\mathbb{R}^{d}$. submitted, arXiv:1710.07077.

[8] W. Dörfler, A. Lechleiter, M. Plum, G. Schneider, and C. Wieners. Photonic Crystals: Mathematical Analysis and Numerical Approximation. Oberwolfach Seminars. Birkhäuser Verlag, Basel, 2011.

[9] J. Giannoulis, A. Mielke, and Ch. Sparber. Interaction of modulated pulses in the nonlinear Schrödinger equation with periodic potential. J. Differential Equations, 245(4):939-963, 2008.

[10] R. H. Goodman, M. I. Weinstein, and P. J. Holmes. Nonlinear propagation of light in one-dimensional periodic structures. J. Nonlin. Sci., 11(2):123-168, 2001.

[11] R. Hammer. Dynamics of Dirac fermions. PhD thesis, Graz U., 2013.

[12] L. Hörmander. The Analysis of Linear Partial Differential Operators III: Pseudo-Differential Operators. A Series of Comprehensive Studies in Mathematics / Grundlehren Der Mathematischen Wissenschaften. Springer Berlin Heidelberg, 1985.

[13] T. Katō. Perturbation theory for linear operators. Grundlehren der mathematischen Wissenschaften. Springer, Berlin, 1995. 
[14] D.E. Pelinovsky. Localization in Periodic Potentials: From Schrödinger Operators to the Gross-Pitaevskii Equation. London Mathematical Society Lecture Note Series. Cambridge University Press, 2011.

[15] V. I. Petviashvili. Equation of an extraordinary soliton. Plasma Physics, 2:469, 1976.

[16] G. Schneider and H. Uecker. Nonlinear coupled mode dynamics in hyperbolic and parabolic periodically structured spatially extended systems. Asymptot. Anal., 28(2):163-180, 2001.

1 Martin-Luther-Universität Halle-Wittenberg, Institut für Mathematik, D-06099 Halle (Saale), Germany; ${ }^{2}$ Technische Universität Dortmund, Fakultät für Mathematik, VogelpothSweg 87, D-44227 Dortmund, Germany

E-mail address: tomas.dohnal@mathematik.uni-halle.de, lisa.wahlers@math.tu-dortmund.de 